\title{
RELATIVE COMPLETE REDUCIBILITY AND NORMALIZED SUBGROUPS
}

\author{
MAIKE GRUCHOT ${ }^{1}$, ALASTAIR LITTERICK $^{\circledR 2}$ and GERHARD RÖHRLE ${ }^{3}$ \\ ${ }^{1}$ Lehrstuhl für Algebra und Zahlentheorie, RWTH Aachen University, D-52062 Aachen, Germany; \\ email: maike.gruchot@rwth-aachen.de \\ ${ }^{2}$ Department of Mathematical Sciences, University of Essex, Wivenhoe Park, Colchester, \\ Essex CO4 3SQ, UK; \\ email: a.litterick@essex.ac.uk \\ ${ }^{3}$ Fakultät für Mathematik, Ruhr-Universität Bochum, Universitätsstraße 150, \\ D-44780 Bochum, Germany; \\ email: gerhard.roehrle@rub.de
}

Received 19 November 2019; accepted 27 April 2020

\begin{abstract}
We study a relative variant of Serre's notion of $G$-complete reducibility for a reductive algebraic group $G$. We let $K$ be a reductive subgroup of $G$, and consider subgroups of $G$ that normalize the identity component $K^{\circ}$. We show that such a subgroup is relatively $G$-completely reducible with respect to $K$ if and only if its image in the automorphism group of $K^{\circ}$ is completely reducible. This allows us to generalize a number of fundamental results from the absolute to the relative setting. We also derive analogous results for Lie subalgebras of the Lie algebra of $G$, as well as 'rational' versions over nonalgebraically closed fields.
\end{abstract}

2010 Mathematics Subject Classification: 20G15 (primary); 14L24 (secondary)

\section{Introduction}

Let $G$ be a (possibly disconnected) reductive algebraic group over an algebraically closed field. In recent years, much effort has been devoted to understanding Serre's notion of $G$-complete reducibility $[1,4-7,12,13,17,18,20]$. This powerful notion links the subgroup structure of $G$ with representation theory (which can be viewed as the special case $G=\mathrm{GL}_{n}$ ) and with concepts from geometric invariant theory.

(C) The Author(s) 2020. This is an Open Access article, distributed under the terms of the Creative Commons Attribution licence (http://creativecommons.org/licenses/by/4.0/), which permits unrestricted re-use, distribution, and reproduction in any medium, provided the original work is properly cited. 
In [7], a relative version of this concept is introduced. If $K$ is a closed reductive subgroup of $G$, then a closed subgroup $H$ of $G$ is called relatively $G$-completely reducible with respect to $K$ if, whenever $H$ is contained in an R-parabolic subgroup $P_{\lambda}$ for a cocharacter $\lambda$ of $K$, then $H$ is contained in an R-Levi subgroup $L_{\mu}$ for some cocharacter $\mu$ of $K$ with $P_{\lambda}=P_{\mu}$. More detailed definitions are given in Section 2.

Our main result is a direct relation between this relative variant and the usual 'absolute' notion of complete reducibility (which is the case $K=G$ ) when considering subgroups that normalize the identity component $K^{\circ}$ of $K$. This allows us to deduce relative versions of many pivotal theorems directly from their absolute counterparts, which we do in Section 4. Throughout, all groups are defined over a fixed field $k$ which, until Section 7, is taken to be algebraically closed.

THEOREM 1. Let $K \leqslant G$ be reductive algebraic groups, write $N=N_{G}\left(K^{\circ}\right)$, $C=C_{G}\left(K^{\circ}\right)$, and let $\pi: N \rightarrow N / C$ be the quotient map. Let $H$ be a closed subgroup of $N$. Then $H$ is relatively $G$-completely reducible with respect to $K$ if and only if $\pi(H)$ is $\pi(N)$-completely reducible.

Note that we do not assume that any of the groups $G, H, K, N$ or $C$ in Theorem 1 is connected. Since $K^{\circ}$ is reductive, $\pi(N)$ is a finite extension of the connected semisimple group $\operatorname{Inn}\left(K^{\circ}\right) \cong K^{\circ} / Z\left(K^{\circ}\right)$. Hence it is reductive and $\pi(N)$-complete reducibility makes sense; cf. [4, Section 6].

The assumption that $H$ normalizes $K^{\circ}$ is a natural one. It holds in the absolute case or if $H$ is contained in $K$, and is often necessary to obtain sensible statements for relative results. More importantly, many natural extensions of results fail without this assumption, as we now illustrate. Recall that a $G$ completely reducible subgroup is necessarily reductive [17, Property 4], and the converse to this holds in characteristic zero [18, Théorème 4.4]. However, neither direction holds in full generality in the relative setting, as pointed out in [7, Remarks 3.2]. Nevertheless, the following consequence of Theorem 1 faithfully generalizes this to the relative setting, as long as $H$ normalizes $K^{\circ}$. We discuss this further in Section 4.

COROLlaRY 2. Let $K \leqslant G$ be reductive algebraic groups and let $H \leqslant N_{G}\left(K^{\circ}\right)$. If $H$ is relatively $G$-completely reducible with respect to $K$, then the unipotent radical $R_{u}(H)$ centralizes $K^{\circ}$. Conversely, if $k$ has characteristic zero and $R_{u}(H)$ centralizes $K^{\circ}$, then $H$ is relatively $G$-completely reducible with respect to $K$. 
As in the absolute case, the reverse direction of Corollary 2 also holds if the characteristic char $(k)$ of $k$ is sufficiently large relative to $K$ and $H$ (independently of $G$ ), and this specializes to [18, Théorème 4.4] in the case $K=G$. A more detailed statement is given in Theorem 4.2.

Just as $G$-complete reducibility can be expressed in terms of the closure of orbits of $G$ on its Cartesian products $G^{n}$ under conjugation [4, Corollary 3.7], the relative notion can be characterized in terms of the closure of orbits of $K$ on $G^{n}$ (see Theorem 2.1). Since $\pi(N)^{\circ}=\pi\left(K^{\circ}\right)$ and an orbit $K \cdot \mathbf{h}$ is closed in $G^{n}$ if and only if $K^{\circ} \cdot \mathbf{h}$ is closed in $G^{n}$, Theorem 1 is equivalent to the following.

THEOREM 3. Let $K \leqslant G$ be reductive algebraic groups, write $N=N_{G}\left(K^{\circ}\right)$, $C=C_{G}\left(K^{\circ}\right)$, and let $\pi: N \rightarrow N / C$ be the quotient map. Let $\mathbf{h} \in N^{n}(n \geqslant 1)$, and write $\pi$ also for the induced map $N^{n} \rightarrow(N / C)^{n}$. Then $K \cdot \mathbf{h}$ is closed in $G^{n}$ if and only if $\pi(N) \cdot \pi(\mathbf{h})$ is closed in $(N / C)^{n}$.

In the absolute case, $G$-complete reducibility of a subgroup $H$ interacts with separability of $H$ in $G$ [4, Definition 3.27] and semisimplicity of the Lie algebra $\operatorname{Lie}(G)$ as an $H$-module $[6,21]$. In Section 5, we define a relative version of separability, and show that the corresponding results still hold. The next two results are particularly interesting.

The following is a generalization of [6, Theorem 1.7] both to the relative setting and to the case that $G$ may not be connected. Recall that a prime $p$ is called bad for the reductive group $G$ if $p$ divides some coefficient when the highest root in the root system of some simple factor of $G$ is expressed as a sum of simple roots. The characteristic char $(k)$ of $k$ is called $\operatorname{good}$ for $G$ if it is not a bad prime. If $\operatorname{char}(k)$ is good and not a divisor of $r+1$ whenever $G$ has a simple factor $A_{r}$, then it is called very good for $G$.

THEOREM 4. Let $K \leqslant G$ be reductive algebraic groups, write $N=N_{G}\left(K^{\circ}\right)$, $C=C_{G}\left(K^{\circ}\right)$, and let $\pi: N \rightarrow N / C$ be the quotient map. Suppose that $\operatorname{char}(k)$ is zero or is very good for $K$ and does not divide $\left|\pi(N) / \pi\left(N^{\circ}\right)\right|$. If $H \leqslant N$ and $\operatorname{Lie}(K)$ is semisimple as an $H$-module, then $H$ is relatively $G$-completely reducible with respect to $K$.

Theorem 4 in fact holds under a slightly weaker condition on char $(k)$ involving $H$ as well as $K$ and $N_{G}\left(K^{\circ}\right)$; see Corollary 5.5.

Next, for subgroups $H$ and $K$ of an algebraic group $G$, we say that $(G, K)$ is a reductive pair for $H$ if $\operatorname{Lie}(K)$ is an $H$-module direct summand of $\operatorname{Lie}(G)$ (Definition 3 ). The interplay between this notion and separability (Lemma 5.6) gives a further condition for $G$-complete reducibility (Corollary 5.7). Due to 
the well-known fact that every subgroup of $\operatorname{GL}(V)$ is separable, we obtain a particularly nice criterion in this case, with no dependence on $\operatorname{char}(k)$. This is orthogonal to other criteria derived recently in [1]. The proof can be found at the end of Section 5.

Theorem 5. Let $G=\mathrm{GL}(V)$, let $K \leqslant G$ be reductive and let $H \leqslant N_{G}\left(K^{\circ}\right)$. Suppose that $(G, K)$ is a reductive pair for $H$. If $\operatorname{Lie}(K)$ is semisimple as an $H$-module, then $H$ is relatively $G$-completely reducible with respect to $K$.

For general connected $G$, we obtain the following criterion on $\operatorname{char}(k)$ for complete reducibility, which depends on $G$ but not on $K, N_{G}\left(K^{\circ}\right)$ or $H$.

THEOREM 6. Maintain the notation of Theorem 1 and suppose that $G$ is connected. Suppose that $(G, K)$ is a reductive pair for $H$ and that $\operatorname{char}(k)$ is very good for $G$. If $\mathrm{Lie}(K)$ is semisimple as an $H$-module, then $H$ is relatively $G$-completely reducible with respect to $K$.

The paper is organized as follows. After recalling relevant background in Section 2, we prove Theorem 1 in Section 3. We also briefly discuss the analogue of Theorem 1 for relative $G$-irreducibility (Corollary 3.9). In Section 4, we derive a series of consequences of Theorem 1, including Corollary 2. In Section 5, we introduce our relative notions of separability and reductive pairs, and generalize further results from the absolute case, particularly from [6]. In Section 6, we consider relative $G$-complete reducibility of Lie subalgebras of $\operatorname{Lie}(G)$, and derive variants of Theorems 1 and 3. Finally, in Section 7, we consider a rational version of relative $G$-complete reducibility, dropping the assumption that $k$ is algebraically closed. Again, variants of Theorems 1 and 3 hold in this setting.

\section{Notation and preliminaries}

Throughout, $k$ denotes a field and $\operatorname{char}(k)$ denotes the characteristic of $k$. We take $k$ to be algebraically closed, until Section 7, where we generalize our main results. All groups encountered are affine algebraic groups, meaning Zariskiclosed subgroups of some general linear group over $k$ (or its algebraic closure $\bar{k}$ in Section 7). Homomorphisms between groups are morphisms of varieties, and subgroups are closed. For an algebraic group $G$, we denote by $G^{\circ}$ the connected component of the identity element. For a subgroup $K$ of $G$, the normalizer and centralizer of $K$ in $G$ are, respectively, denoted by $N_{G}(K)$ and $C_{G}(K)$. The unipotent radical of $G$, denoted by $R_{u}(G)$, is the (unique) maximal connected normal unipotent subgroup of $G$. We say that $G$ is reductive if $R_{u}(G)=1$. 
We do not require that a reductive group is connected. An isogeny means a surjective map $G_{1} \rightarrow G_{2}$ with finite kernel, where $G_{1}$ and $G_{2}$ are reductive. In this case, the kernel centralizes $G_{1}^{\circ}$.

A cocharacter of $G$ is a homomorphism of algebraic groups from the multiplicative group $k^{*}$ to $G$. The set of all cocharacters of $G$ is denoted by $Y(G)$. Conjugation induces an action of $G$ on $Y(G)$, with $(g \cdot \phi)(c) \stackrel{\text { def }}{=} g \phi(c) g^{-1}$ for all $g \in G, \phi \in Y(G), c \in k^{*}$. We also use a dot to denote left conjugation of $G$ on itself and on the Cartesian products $G^{n}$. If $\phi: k^{*} \rightarrow G$ is a morphism, we say that the $\operatorname{limit}_{a \rightarrow 0} \phi(a)$ exists if there is a morphism $\widehat{\phi}: k \rightarrow G$ whose restriction to $k^{*}$ is $\phi$. In this case, we write $\lim _{a \rightarrow 0} \phi(a)$ for $\widehat{\phi}(0)$. If the limit exists, then it is unique, as $k^{*}$ is Zariski-dense in $k$. For $\lambda \in Y(G)$, define the following subgroups of $G$ :

$$
\begin{aligned}
& P_{\lambda} \stackrel{\text { def }}{=}\left\{g \in G: \lim _{a \rightarrow 0}(\lambda(a) \cdot g) \text { exists }\right\}, \\
& L_{\lambda} \stackrel{\text { def }}{=}\left\{g \in G: \lim _{a \rightarrow 0}(\lambda(a) \cdot g)=g\right\}=C_{G}\left(\lambda\left(k^{*}\right)\right) .
\end{aligned}
$$

Following [4], such a subgroup $P_{\lambda}$ is called a Richardson parabolic subgroup, or an R-parabolic subgroup of $G$, and $L_{\lambda}$ is called a Richardson Levi subgroup of $G$, or an R-Levi subgroup. If $G$ is connected reductive, then these definitions coincide with the usual definitions of parabolic subgroups and Levi subgroups [4, Section 6]. The unipotent radical of $P_{\lambda}$ is given by

$$
R_{u}\left(P_{\lambda}\right)=\left\{g \in G: \lim _{a \rightarrow 0}(\lambda(a) \cdot g)=1\right\} .
$$

If $K$ is a reductive subgroup of $G$, then the inclusion $K \rightarrow G$ induces an injective map $Y(K) \rightarrow Y(G)$, and we identify $Y(K)$ with its image in $Y(G)$. The following is the central notion of the paper.

DEFINITION 1. Let $H$ and $K$ be closed subgroups of an algebraic group $G$. We say that $H$ is relatively $G$-completely reducible with respect to $K$ if, for every cocharacter $\lambda \in Y(K)$ such that $H \leqslant P_{\lambda}$, there exists a cocharacter $\mu \in Y(K)$ such that $P_{\lambda}=P_{\mu}$ and $H \leqslant L_{\mu}$.

While the above definitions make sense without assumptions on $G$ or $K$, we always assume that both $K$ and $G$ are reductive. We refer to the case $K=G$ as the absolute case, and say that $H$ is $G$-completely reducible if the above holds in this case. For brevity, we sometimes write 'relatively $G$-cr with respect to $K$ ', or just ' $G$-cr' in the absolute case.

Recall from [8, Definition 5.4] that a generic tuple for a subgroup $H \leqslant G$ is an $n$-tuple $\mathbf{h} \in G^{n}(n \geqslant 1)$ such that the elements in $\mathbf{h}$ generate the same associative 
$k$-subalgebra of $\operatorname{Mat}_{m \times m}(k)$ as $H$, for some faithful representation $G \rightarrow \mathrm{GL}_{m}(k)$. For instance, a tuple of elements that topologically generate $H$ is a generic tuple for $H$ [8, Remark 5.6].

TheOREM 2.1 [7, Theorem 1.1]. Let $K \leqslant G$ be reductive algebraic groups, let $H$ be a closed subgroup of $G$ and let $\mathbf{h} \in G^{n}$ be a generic tuple for $H$. Then $H$ is relatively $G$-completely reducible with respect to $K$ if and only if the orbit $K \cdot \mathbf{h}$ is closed in $G^{n}$.

REMARK 2.2. Let $H$ be a subgroup of $G$ and let $\mathbf{h} \in G^{n}$ be a generic tuple for $H$. Then we can lengthen $\mathbf{h}$ so that $\pi(\mathbf{h})$ is also a generic tuple for $\pi(H)$. Together with Theorem 2.1, this shows that Theorem 3 implies Theorem 1. Conversely, given $\mathbf{h} \in G^{n}$, we let $H$ be the closed subgroup topologically generated by the elements of $\mathbf{h}$. Then $\pi(H)$ is topologically generated by $\pi(\mathbf{h})$ and it follows at once that Theorem 1 implies Theorem 3.

REMARK 2.3. Although our methods make intrinsic use of the fact that $K$ is reductive, Theorem 2.1 shows that the assumption that $G$ is reductive is unimportant in the relative setting, since we are free to replace $G$ with any closed subgroup of $G$ containing $H$ and $K$, or with any group $G^{\prime}$ containing $G$ as a closed subgroup; cf. [7, Corollary 3.6]. Although it is beyond the scope of this paper, there is the potential to develop the theory of relative complete reducibility with respect to arbitrary closed subgroups $K$. For instance, applying Definition 1 in the case when $Y(K)$ is trivial, that is, when $K^{\circ}$ is unipotent, we see that every subgroup is relatively $G$-cr with respect to $K$. This corresponds to the geometric fact that all orbits of a unipotent group on a variety are closed.

\section{Proof of Theorem 1}

Recall our set-up that $G$ is a (not necessarily connected) reductive algebraic group, and $H$ and $K$ are closed subgroups of $G$ with $K$ reductive. Write $N=$ $N_{G}\left(K^{\circ}\right), C=C_{G}\left(K^{\circ}\right)$, let $\pi: N \rightarrow N / C$ be the quotient map, and assume that $H \leqslant N$. We begin with a crucial lemma. This is straightforward if $N$ is reductive; cf. [4, Lemma 6.14]. But see Remark 3.2 for a subtle point, which arises when $N$ has a nontrivial unipotent radical.

LEMMA 3.1. In the setting of Theorem 1, there exists a reductive subgroup $M$ of $N$ such that

(1) $M^{\circ}=\left[K^{\circ}, K^{\circ}\right], M \cap C$ is finite and $N=M C$; 
(2) the restriction of $\pi$ to $M$ gives an isogeny $M \rightarrow N / C$;

(3) for all $\lambda \in Y(K)$, we have $P_{\lambda} \cap N=\left(P_{\lambda} \cap M\right) C$ and $L_{\lambda} \cap N=\left(L_{\lambda} \cap M\right) C$.

Proof. Note first that $N^{\circ}=\left[K^{\circ}, K^{\circ}\right] C^{\circ}$ since $N / C$ is a finite extension of $\operatorname{Inn}\left(K^{\circ}\right)=K^{\circ} / Z\left(K^{\circ}\right)=\left[K^{\circ}, K^{\circ}\right] / Z\left(\left[K^{\circ}, K^{\circ}\right]\right)$. Consider the quotient map $\sigma: N \rightarrow N /\left[K^{\circ}, K^{\circ}\right]$. By the above, $N /\left[K^{\circ}, K^{\circ}\right]$ is a finite extension of $\sigma\left(\left[K^{\circ}, K^{\circ}\right] C\right)$. By [10, Theorem 1.1], $N /\left[K^{\circ}, K^{\circ}\right]$ admits a finite subgroup $F$ such that $N /\left[K^{\circ}, K^{\circ}\right]=F\left(\sigma\left(\left[K^{\circ}, K^{\circ}\right] C\right)\right)$. Let $M$ be the preimage of $F$ under $\sigma$. Then $M^{\circ}=\left[K^{\circ}, K^{\circ}\right]$ by construction, and $M \cap C$ is finite since it is a finite extension of $\left[K^{\circ}, K^{\circ}\right] \cap C$, which is itself finite as it is contained in $Z\left(\left[K^{\circ}, K^{\circ}\right]\right)$. Finally, $N=\sigma^{-1}\left(F \sigma\left(\left[K^{\circ}, K^{\circ}\right] C\right)\right)=M \sigma^{-1}(\sigma(C)) \leqslant M C$; hence $N=M C$. Thus (1) is proved. Now (2) and (3) follow directly from the fact that $N=M C$, $M \cap C$ is finite and $C \leqslant L_{\lambda} \leqslant P_{\lambda}$ for all $\lambda \in Y(K)$.

REMARK 3.2. If $N$ is reductive, then [4, Lemma 6.14] lets us take $M^{\circ}$ to be the product of the simple factors of $N^{\circ}$, which commute with $C^{\circ}$ (which is also reductive in this case). The following example shows that in general $M$ need not be isomorphic to its image in the reductive group $N / R_{u}(C)$; in particular, $M$ could be a nonsplit extension of $M \cap R_{u}(C)$.

Let $K=\mathrm{SL}_{3}(k)$, where $\operatorname{char}(k)=2$, and let $C$ be a copy of $\mathbb{G}_{a}$. Let $X=\langle x\rangle$ be cyclic of order 4 , and consider the product $(X \times C) /\left\langle x^{2} y\right\rangle$ for any nonzero $y \in k$. Write $H$ for the resulting Abelian two-fold nonsplit extension of $C$, so $H^{\circ}=C$.

Now let $H$ act on $K$, such that $C$ acts trivially and $H / C$ acts as the inversetranspose automorphism, and write $G=N$ for the semidirect product $K \rtimes H$. By construction, $K=K^{\circ}$ is normal in $N$, and $C=H^{\circ}=C_{N}\left(K^{\circ}\right)$ is connected and unipotent. In $N / C$, the subgroup $M$ guaranteed by [4, Lemma 6.14] is the image of the outer automorphism, that is, there is an involution giving a complement to $K=(N / C)^{\circ}$. But in $N$, this element has order 4 by construction. So the smallest $M$ satisfying the conclusion of Lemma 3.1 is a four-fold extension $\mathrm{SL}_{3}(k) \rtimes\langle x\rangle$, where $x$ acts as the inverse-transpose automorphism on $\mathrm{SL}_{3}(k)$; this is a nonsplit extension of $M \cap C=\left\langle x^{2}\right\rangle$.

Proof of Theorem 1. Let $G, K, H, N=N_{G}\left(K^{\circ}\right), C=C_{G}\left(K^{\circ}\right)$ and $\pi: N \rightarrow$ $N / C$ be as in Theorem 1, and let $M$ be the reductive subgroup given by Lemma 3.1.

To begin, for $\lambda \in Y(K)$, we have $C \leqslant L_{\lambda} \leqslant P_{\lambda}$. Hence $H C$ is contained in $P_{\lambda}$ (respectively, $L_{\lambda}$ ) if and only if $H$ is contained in $P_{\lambda}$ (respectively, $L_{\lambda}$ ). Moreover, since $N=M C$, we have $H C=(H C \cap M) C$, and $H C$ is contained in $P_{\lambda}$ (respectively, $L_{\lambda}$ ) if and only if $H C \cap M$ is contained in $P_{\lambda}$ (respectively, $L_{\lambda}$ ). Since also $\pi(H)=\pi(H C)=\pi(H C \cap M)$, it suffices to prove the conclusion of Theorem 1 for subgroups of the form $H C \cap M$, in particular for subgroups of $M$. 
So assume $H \leqslant M$. Then the reductive subgroup $M Z\left(K^{\circ}\right)^{\circ}$ contains both $K^{\circ}$ and $H$. Since also $Y(K)=Y\left(K^{\circ}\right)$, as in Remark 2.3, it follows that $H$ is relatively $G$-cr with respect to $K$ if and only if $H$ is relatively $M Z\left(K^{\circ}\right)^{\circ}$-cr with respect to $K^{\circ}$. Moreover, because $\pi\left(M Z\left(K^{\circ}\right)^{\circ}\right)=\pi(M)=\pi(N)$, it suffices to prove the conclusion of Theorem 1 when $G=N=M Z\left(K^{\circ}\right)^{\circ}$, a reductive group in which $C^{\circ}=C_{G}\left(K^{\circ}\right)^{\circ}$ is a central torus of $G^{\circ}$. We can still assume $H \leqslant M$.

Now, under these assumptions, we have $Y(G)=Y(K)$; so $H$ is relatively $G$ cr with respect to $K$ if and only if $H$ is $G$-cr. In this situation, the statement of Theorem 1 becomes: 'Let $G$ be reductive and let $K$ be a reductive subgroup of $G$ such that $K^{\circ}$ is normal in $G$. Let $\pi: G \rightarrow G / C_{G}\left(K^{\circ}\right)$, where $C_{G}\left(K^{\circ}\right)^{\circ}$ is a central torus of $G^{\circ}$. Then $H$ is $G$-cr if and only if $\pi(H)$ is $\left(G / C_{G}\left(K^{\circ}\right)\right)$-cr.' This is proved in [4, Section 6.2]. More specifically, the result for connected groups is part of [4, Lemma 2.12(ii)(b)], and [4, Section 6.2] generalizes this part to nonconnected reductive groups.

REMARK 3.3. After the reduction to the case $G=N$, the above proof of Theorem 1 is similar to the argument of [5, Theorem 3.4], which shows that the $G$ complete reducibility of a subgroup $H \leqslant G$ is preserved under taking quotients by subgroups of $H$, which are normal in $G$. Indeed, adapting that argument provides another proof of Theorem 1, where one first reduces to the case $C \leqslant H$ (rather than $H \leqslant M$ ).

REMARK 3.4. If instead of $H \leqslant N$, we assume that both $H$ and $K$ are reductive and $K^{\circ}$ normalizes $H$, then $H$ is always relatively $G$-cr with respect to $K$. This follows from [7, Corollary 3.28] since if $K$ normalizes $H$, then in particular a maximal torus of $K$ normalizes $H$, and this implies that $H$ is relatively $G$-cr with respect to $K$.

Before discussing a number of consequences of Theorem 1 in the next section, we take this opportunity to note a slightly more general result and to observe that some subtleties can arise when dealing with R-parabolic and R-Levi subgroups of disconnected groups. The following slight generalization of Theorem 1 shows that we can factor out any normal subgroup of $C_{G}\left(K^{\circ}\right)$ without affecting relative complete reducibility.

THEOREM 3.5. Keep the notation of Theorem 1 and let $M$ be as in Lemma 3.1. Let $f: N \rightarrow G^{\prime}$ be a homomorphism into a reductive group $G^{\prime}$ such that $\operatorname{ker}(f) \leqslant C$. If $H \leqslant M \operatorname{ker}(f)$, then $H$ is relatively $G$-completely reducible with respect to $K$ if and only if $f(H)$ is relatively $G^{\prime}$-completely reducible with respect to $f(K)$. 
Proof. As in the proof of Theorem 1, it suffices to prove the result for the subgroup $H \operatorname{ker}(f) \cap M$ of $M$, because this is contained in an R-parabolic or RLevi subgroup corresponding to $\lambda \in Y(K)$ if and only if $H$ is since $\operatorname{ker}(f) \leqslant C$. Moreover, the hypotheses imply that $f(H \operatorname{ker}(f) \cap M)=f(H)$. Also, since $\operatorname{ker}(f) \leqslant C$, and since the restriction $\pi: M \rightarrow N / C$ gives an isogeny onto its image, the restriction $f: M \rightarrow N / \operatorname{ker}(f)$ is also an isogeny onto its image. Then as before, we can reduce to the case that $G=N=M Z\left(K^{\circ}\right)^{\circ}$, since this contains both $K^{\circ}$ and $H$, and this does not change $f(H)$ or the set of cocharacters of $f(K)$, and hence does not change which R-parabolic subgroups or R-Levi subgroups of $G^{\prime}$ stemming from $f(K)$ contain $f(H)$. Thus $f(G)$ is reductive and contains $f(K)$ and $f(H)$, and we can thus also assume that $f(G)=G^{\prime}$.

So now $H$ is relatively $G$-cr with respect to $K^{\circ}$ precisely when $H$ is $G$-cr, and also $f\left(K^{\circ}\right)=\left(G^{\prime}\right)^{\circ}$; so $f(H)$ is relatively $G^{\prime}$-cr with respect to $f(K)$ precisely when $f(H)$ is $G^{\prime}$-cr. As before, the desired result reduces to the result proved in $[4$, Section 6.2].

REMARK 3.6. When $\lambda \in Y(K) \backslash Y(M)$, it is not necessarily the case that $\pi\left(P_{\lambda} \cap N\right)=P_{\pi \circ \lambda}$ and $\pi\left(L_{\lambda} \cap N\right)=L_{\pi \circ \lambda}$, as the following example shows. So although relative complete reducibility behaves well with respect to taking quotients by subgroups centralizing $K^{\circ}$, some care is required in the proofs.

Let $G$ be a connected reductive group with a maximal torus $T$ such that $T$ and $N_{G}(T) / T$ are nontrivial, and let $K=N=N_{G}(T)$. Then $K^{\circ}=C=T$ and $\pi(N)=N / C$ is the Weyl group of $G$, and hence finite. For the subgroup $M$ of Lemma 3.1, we can take any finite subgroup of $K$, which maps onto $\pi(N)$. Then $Y(M)=Y\left(\left[K^{\circ}, K^{\circ}\right]\right)$ contains only the trivial cocharacter. Thus the only R-parabolic subgroup (and the only R-Levi subgroup) of $\pi(N)$ is $\pi(N)$ itself, and its preimage under $\pi$, namely $N$, is not contained in any proper R-parabolic subgroup of $G$. Thus for any $\lambda \in Y(K)$ whose image is noncentral in $G$, the images $\pi\left(P_{\lambda} \cap N\right)$ and $\pi\left(L_{\lambda} \cap N\right)$ are proper subgroups of $\pi(N)$ and are therefore not R-parabolic subgroups or R-Levi subgroups.

REMARK 3.7. Recall that a subgroup $H$ of $G$ is called relatively $G$-irreducible with respect to $K$ if, whenever $\lambda \in Y(K)$ such that $H \leqslant P_{\lambda}$, we have $P_{\lambda}=G$ [7, Definition 3.14]. In this case, if $K=G$, then $H$ is called $G$-irreducible [18]. We observe that the analogue of Theorem 1 does not hold for relative $G$ irreducibility. For instance, if $K$ contains any noncentral torus $S$ of $G$, then $C$ is contained in $C_{G}(S)$, a proper R-Levi subgroup of $G$, so $C$ is not relatively $G$-irreducible with respect to $K$. On the other hand, if we suppose that 
$\pi(N)=N / C$ is finite, then every subgroup of $\pi(N)$ is $\pi(N)$-irreducible since $Y(\pi(N))$ is trivial; in particular, the trivial subgroup (which is the image of $C)$ is $\pi(N)$-irreducible in this case.

The essential problem in the above discussion is the existence of nontrivial tori in the kernel of $N \rightarrow N / C$, that is, a nontrivial torus in $Z\left(K^{\circ}\right)$. The following result generalizes [4, Lemma 6.15], which considers the case that $N$ is reductive. This allows us to extend Theorem 1 to relative $G$-irreducibility, assuming $K^{\circ}$ is semisimple (Corollary 3.9).

Proposition 3.8. Keep the notation of Theorem 1 and let $M \leqslant N$ be a reductive subgroup guaranteed by Lemma 3.1 so that $N=M C$ and $M \cap C$ is finite. Let $f: N \rightarrow G^{\prime}$ be a homomorphism into a reductive group $G^{\prime}$ such that $\operatorname{ker}(f) \leqslant C$. If $\lambda \in Y(M)$, then

$$
\begin{gathered}
f\left(P_{\lambda} \cap N\right)=f\left(P_{\lambda} \cap M\right) f(C)=P_{f \circ \lambda} \cap f(N), \\
f^{-1}\left(P_{f \circ \lambda} \cap f(N)\right)=P_{\lambda} \cap N, \\
f\left(L_{\lambda} \cap N\right)=f\left(L_{\lambda} \cap M\right) f(C)=L_{f \circ \lambda} \cap f(N), \\
f^{-1}\left(L_{f \circ \lambda} \cap f(N)\right)=L_{\lambda} \cap N .
\end{gathered}
$$

In particular, taking $f=\pi: N \rightarrow N / C$, we have

$$
\begin{aligned}
& \pi\left(P_{\lambda} \cap N\right)=\pi\left(P_{\lambda} \cap M\right)=P_{\pi \circ \lambda}, \quad \pi^{-1}\left(P_{\pi \circ \lambda}\right)=P_{\lambda} \cap N, \\
& \pi\left(L_{\lambda} \cap N\right)=\pi\left(L_{\lambda} \cap M\right)=L_{\pi \circ \lambda}, \quad \pi^{-1}\left(L_{\pi \circ \lambda}\right)=L_{\lambda} \cap N .
\end{aligned}
$$

Proof. Since $\operatorname{ker}(f) \leqslant C$ and $\pi: M \rightarrow N / C$ is an isogeny onto its image, it follows that $f: M \rightarrow f(M)$ is an isogeny. Thus by the nonconnected version of [4, Lemma 2.11], the R-parabolic subgroups and R-Levi subgroups of $f(M)$ are precisely the subgroups $P_{f \circ \lambda} \cap f(M)$ and $L_{f \circ \lambda} \cap f(M)$ for $\lambda \in Y(M)$, and these are, respectively, equal to $f\left(P_{\lambda} \cap M\right)$ and $f\left(L_{\lambda} \cap M\right)$. Since $C \leqslant L_{\lambda} \leqslant P_{\lambda}$ for all $\lambda \in Y(K)$, we also have $f(C) \leqslant L_{f \circ \lambda} \leqslant P_{f \circ \lambda}$, and since also $N=M C$, we have $f(N)=f(M) f(C)$ and it follows that

$$
\begin{aligned}
f\left(P_{\lambda} \cap N\right) & =f\left(\left(P_{\lambda} \cap M\right) C\right)=f\left(P_{\lambda} \cap M\right) f(C) \\
& =\left(P_{f \circ \lambda} \cap f(M)\right) f(C)=P_{f \circ \lambda} \cap f(N),
\end{aligned}
$$

and $f\left(L_{\lambda} \cap N\right)=f\left(L_{\lambda} \cap M\right) f(C)=L_{f \circ \lambda} \cap f(N)$ follows similarly. Finally, since $\operatorname{ker}(f) \leqslant C$, we have

$$
f^{-1}\left(P_{f \circ \lambda} \cap f(N)\right)=f^{-1} f\left(P_{\lambda} \cap N\right)=\left(P_{\lambda} \cap N\right) \operatorname{ker}(f)=P_{\lambda} \cap N
$$

and similarly for $L_{\lambda}$. 
The later statements above show in particular that if $H$ is a subgroup of $G$, then $H \leqslant P_{\lambda}$ or $L_{\lambda}$ for $\lambda \in Y(M)$ precisely when $\pi(H) \leqslant P_{\pi \circ \lambda}$ or $L_{\pi \circ \lambda}$, respectively. In particular, if $Y(K)=Y(M)$ (that is if $K^{\circ}$ is semisimple), we obtain the following.

COROLlaRY 3.9. In the notation of Theorem 1 , if $K^{\circ}$ is semisimple, then a subgroup $H$ of $N$ is relatively $G$-irreducible with respect to $K$ if and only if $\pi(H)$ is $\pi(N)$-irreducible.

We close this section with an extended example illustrating Theorem 1.

EXAMPLE 3.10. Write $K=C l(W)$ to indicate that $K$ is a special orthogonal or symplectic group with natural module $W$, in characteristic $p \geqslant 0$. Take an orthogonal direct sum $V=W_{1} \perp W_{2}$, where $W_{i} \cong W$ for $i=1,2$, and let $G=$ $C l(V)$ (so $G$ has type $C_{n}$ or $D_{n}$ for some $n>0$ ). We have a chain of subgroups

$$
K \leqslant C l\left(W_{1}\right) \times C l\left(W_{2}\right) \leqslant C l(V)=G,
$$

where the first embedding is just the diagonal one. Write $K_{0}$ for the left-hand-side group, and $K_{i}=C l\left(W_{i}\right)$ for $i=1,2$.

(1) If $p \neq 2$ then $K_{0}$ is $G$-cr, contained in a Levi subgroup of type $A_{n-1}$ corresponding to a direct-sum decomposition of $V$ into two totally isotropic $K_{0}$-submodules. Thus $C_{G}\left(K_{0}\right)^{\circ}$ is a 1-dimensional torus, consisting of elements acting as a scalar on $W_{1}$ and as the inverse scalar on $W_{2}$.

(2) If $p=2$, then $K_{0}$ stabilizes a unique nonzero totally isotropic subspace of $V$ [4, Example 3.45], which is a diagonal submodule $W_{0} \subset W_{1}+W_{2}$. This shows that $K_{0}$ is contained in a parabolic subgroup of $G$ whose Levi factor has type $A_{n-1}$, but not in any Levi subgroup of $G$. In particular, $K_{0}$ is non- $G$-cr and $C_{G}\left(K_{0}\right)^{\circ}$ is unipotent. In fact, one can show that $C_{G}\left(K_{0}\right)^{\circ}$ is a 1-dimensional unipotent group; writing $V=W_{0}+W_{1}$ and identifying elements of $W_{0}$ and $W_{1}$ via a $K_{0}$-module isomorphism, this unipotent group consists of the maps $\left(w_{0}, w_{1}\right) \mapsto\left(w_{0}+\lambda w_{1}, w_{1}\right)$ for $\lambda \in k$. The image of $K_{0}$ under projection to the Levi factor is a subgroup $K_{0}^{\prime}$ stabilizing a totally isotropic complement to $W_{0}$. Then $K_{0}^{\prime}$ is $G$-cr, and its centralizer is simple of type $A_{1} ; K_{0}^{\prime} C_{G}\left(K_{0}^{\prime}\right)$ acts on $V$ as a tensor product $W_{0} \otimes V_{2}$, where $V_{2}$ is the natural $\mathrm{SL}_{2}(k)$-module.

(3) If we repeat the above construction but with a $K$-module $W_{1} \perp W_{2}$, where $W_{2}$ is nontrivial and not isomorphic to $W_{1}$, then $K_{0}$ is $G$-irreducible and has a trivial connected centralizer (independently of $p$ ). 
These three cases illustrate first that even a uniform (characteristic-independent) construction can lead to variation in $G$-complete reducibility and the structure of normalizers and centralizers. Part (2) gives an example of a nontrivial unipotent subgroup of $G$, namely $C_{G}\left(K_{0}\right)^{\circ}$, which is relatively $G$-cr with respect to $K_{0}$. The tables of [13] give further examples of unipotent groups (and more general groups with nontrivial unipotent radicals) arising as centralizers of reductive groups.

In all three cases, Theorem 1 tells us that if $H \leqslant N_{G}\left(K_{i}\right)$, then $H$ is relatively $G$-cr with respect to $K_{i}$ if and only if the image of $H$ in $K_{i} / Z\left(K_{i}\right)$ is completely reducible. The complete reducibility of this image can be characterized purely in terms of the natural module $W_{i}$ if $i=1$ or 2 . In part (2), $N_{G}\left(K_{0}\right)=K_{0} U_{1}$ is also non- $G$-cr and hence contained in the unique maximal parabolic subgroup of $G$, which contains $K_{0}$; in particular, it stabilizes $W_{0}$. Then a subgroup $H \leqslant N_{G}\left(K_{0}\right)$ acts on $W_{0}$, and to determine whether the image of $H$ in $N_{G}\left(K_{0}\right) / C_{G}\left(K_{0}\right)$ is completely reducible (hence whether $H$ is relatively $G$-cr with respect to $K_{0}$ ), we only need to consider the action of $H$ on $W_{0}$.

\section{Consequences of Theorem 1}

Armed with Theorem 1, we deduce many core results for relative complete reducibility directly from their counterparts in the absolute setting. To begin, keeping the notation of Theorem 1, it is clear that $\pi(N)=N / C$ and the trivial subgroup of $\pi(N)$ are $\pi(N)$-cr. Hence the following is immediate from Theorem 1.

COROLlaRY 4.1. Let $K \leqslant G$ be reductive algebraic groups. Then $N_{G}\left(K^{\circ}\right)$ and $C_{G}\left(K^{\circ}\right)$ are relatively $G$-completely reducible with respect to $K$.

Since $K$ is $K$-cr, that is, relatively $G$-cr with respect to itself, Corollary 4.1 can be viewed as a generalization of [4, Corollaries 3.16 and 3.17], which assert that both $N_{G}\left(K^{\circ}\right)$ and $C_{G}\left(K^{\circ}\right)$ are $G$-cr provided $K$ is. Note that $C_{G}\left(K^{\circ}\right)$ is contained in $L_{\lambda}$ for all $\lambda \in Y(K)$ and is therefore clearly relatively $G$-cr with respect to $K$. However, the conclusion for $N_{G}\left(K^{\circ}\right)$ is less obvious. Note that $N_{G}\left(K^{\circ}\right)$ and $C_{G}\left(K^{\circ}\right)$ need not be reductive in general. When they are reductive, the assertion of Corollary 4.1 follows from [7, Corollary 3.28].

\subsection{Relative complete reducibility, reductivity and semisimple modules.}

As mentioned in Section 1, it is well known that in the absolute case, a $G$-cr subgroup is reductive. Furthermore, a combination of results of Jantzen [11], McNinch [14] and Liebeck and Seitz [12] tells us that if $\operatorname{char}(k)$ is sufficiently large and is coprime to $\left|H / H^{\circ}\right|$ for a closed subgroup $H$ of $G$, then $H$ is $G$-cr if and only if $H$ is reductive. More specifically, for a simple algebraic group $X$ 
define $a(X)$ to be 1 plus the rank of $X$ (the dimension of a maximal torus of $X$ ). For a general reductive group $X$, let $a(X)$ be the maximum value of $a(Y)$ over all simple factors $Y$ of $X$. Then [18, Théorème 4.4] states that if $H$ is a subgroup of $G$, and if $\operatorname{char}(k)$ is zero or a prime $p \geqslant a(G)$ which is coprime to $\left|H / H^{\circ}\right|$, then $H$ is $G$-cr if and only if it is reductive. The relative version of this result, of which Corollary 2 is a special case, is as follows.

THEOREM 4.2. In the notation of Theorem 1, the following hold.

(1) If $H$ is relatively $G$-completely reducible with respect to $K$, then $R_{u}(H) \leqslant C$.

(2) If char $(k)$ is zero or a prime $p \geqslant a(K)$ not dividing $\left|\pi(H) / \pi(H)^{\circ}\right|$, then $H$ is relatively $G$-completely reducible with respect to $K$ if and only if $R_{u}(H) \leqslant C$.

Proof. For (i), Theorem 1 together with the absolute result [17, Property 4] implies that if $H$ is relatively $G$-cr with respect to $K$, then $\pi(H)$ is reductive; hence $R_{u}(H) \leqslant C$. For (ii), if $R_{u}(H) \leqslant C$, then $\pi(H)$ is reductive. Moreover, $\operatorname{char}(k)$ is either zero or coprime to $\left|\pi(H) / \pi(H)^{\circ}\right|$. Then the absolute result [18, Théorème 4.4] tells us that $\pi(H)$ is $\pi(N)$-cr, and from Theorem 1, we see that $H$ is relatively $G$-cr with respect to $K$.

Theorem 4.2 gives an intrinsic group-theoretic characterization of relative $G$-complete reducibility in characteristic zero, generalizing the result from the absolute setting. Next, complete reducibility in the absolute case is closely linked to the semisimplicity of $G$-modules. More precisely, let $T$ be a maximal torus of $G$, let $\Phi^{+}$be a choice of positive roots of $G$ with respect to $T$, and for a $G$ module $V$ define $n(V)=\max \left\{\sum_{\alpha \in \Phi^{+}}\left\langle\lambda, \alpha^{\vee}\right\rangle\right\}$, the maximum over $T$-weights $\lambda$ of $V$. Then a result of Serre [18, Théorème 5.4] states that if $H$ is $G$-cr and char $(k)$ is zero or greater than $n(V)$, then $V$ is semisimple as an $H$-module. Moreover if $V$ is nondegenerate (that is, the identity component of the kernel is a torus), the converse also holds. Since $n(\operatorname{Lie}(G))=2 h_{G}-2$, where $h_{G}$ is the Coxeter number of $G$, this gives a concrete criterion for $G$-complete reducibility in terms of the action of $H$ on $\operatorname{Lie}(G)$ [18, Corollary 5.5]. The relative versions of these results are as follows.

THEOREM 4.3. In the notation of Theorem 1 , the following hold.

(1) Let $V$ be a $\pi(N)$-module, and suppose that char( $k)$ is zero or greater than $n(V)$. If $H$ is relatively $G$-completely reducible with respect to $K$, then $V$ is semisimple as a $\pi(H)$-module. Conversely, if $V$ is nondegenerate and semisimple as a $\pi(H)$-module, then $H$ is relatively $G$-completely reducible with respect to $K$. 
(2) If $\operatorname{char}(k)$ is zero or greater than $2 h_{K}-2$, where $h_{K}$ is the Coxeter number of $K$, then $H$ is relatively $G$-completely reducible with respect to $K$ if and only if $\mathrm{Lie}(K)$ is semisimple as an $H$-module.

Proof. Part (i) follows directly from Theorem 1 and the result in the absolute case applied to $N / C$. For (ii), note that since $N$ normalizes $K^{\circ}$, it acts on the adjoint module $\operatorname{Lie}(K)$; hence so does $H$. Moreover, this action factors through $\pi(N)$ since $C$ centralizes $K^{\circ}$. Now the isomorphism $\pi(N)^{\circ} \cong K^{\circ} / Z\left(K^{\circ}\right)$ implies that $h_{K}=h_{\pi(N)}$. Therefore, since $\operatorname{Lie}(K)$ is nondegenerate as an $(N / C)$-module, part (ii) follows from part (i) and the fact that $n(\operatorname{Lie}(K))=2 h_{K}-2$.

Putting Theorems 4.2(ii) and 4.3(ii) together, and using the fact that $\pi(H) / \pi\left(H^{\circ}\right)$ is a quotient of $H / H^{\circ}$, gives the following.

COROLlaRY 4.4. Let $K \leqslant G$ be reductive algebraic groups, let $H \leqslant N_{G}\left(K^{\circ}\right)$ and suppose that char $(k)$ is zero or a prime $p \geqslant 2 h_{K}-2$ which does not divide $\left|H / H^{\circ}\right|$. Then the following are equivalent:

(1) $H$ is relatively $G$-completely reducible with respect to $K$.

(2) $\operatorname{Lie}(K)$ is semisimple as an $H$-module.

(3) $R_{u}(H)$ centralizes $K^{\circ}$.

A result of Jantzen [11, Proposition 3.2] states that if $G$ is connected and $V$ is a $G$-module, and if $\operatorname{dim} V \leqslant p$ when $\operatorname{char}(k)=p>0$, then $V$ is semisimple. In [2], this is generalized to show that $V$ is also semisimple as an $H$-module for every $G$-cr subgroup $H$ of $G$. The relative variant of this result is as follows; this follows directly from the absolute result [2, Theorem 1.3] and Theorem 1.

COROLlary 4.5. Keep the notation of Theorem 1. Suppose that $\pi(N)$ is connected and let $V$ be a $\pi(N)$-module. If char $(k)=p>0$, assume that $\operatorname{dim} V \leqslant p$.

If $H \leqslant N$ is relatively $G$-completely reducible with respect to $K$, then $V$ is semisimple as an H-module.

The following result also follows directly from its absolute counterpart [2, Theorem 1.4] and Theorem 1. Recall that a module $V$ for an algebraic group is called nondegenerate if the identity component of the kernel of the action is a torus. 
COROLLARY 4.6. With the notation of Theorem 1, suppose that $\pi(N)$ is connected and let $V$ be a nondegenerate $\pi(N)$-module. If $V \otimes V^{*}$ is semisimple as a $\pi(H)$-module, then $H$ is relatively $G$-completely reducible with respect to $K$.

REMARK 4.7. If $V$ is a nondegenerate $\pi(N)$-module, then $V$ is a nondegenerate $K$-module since the identity components of the kernels of $K \rightarrow \pi(K)=$ $K / C_{K}\left(K^{\circ}\right)$ and $\pi(N) \rightarrow \mathrm{GL}(V)$ are both tori. Therefore in the particular case $H \leqslant K$, noting that $H$ is relatively $G$-cr with respect to $K$ precisely when $H$ is $K$-cr, Corollary 4.6 specializes to the complete reducibility statement of [2, Theorem 1.4].

4.2. Normal subgroups, normalizers and Clifford theory. Recall that in the absolute setting, a normal subgroup of a $G$-cr subgroup is again $G$-cr, and furthermore a subgroup $H \leqslant G$ is $G$-cr if and only if $N_{G}(H)$ is $G$-cr [4, Theorem 3.10 and Corollary 3.16]; this generalizes Clifford's theorem from representation theory. Moreover, if $H$ is $G$-cr and $H^{\prime}$ is a subgroup of $G$ that satisfies $H C_{G}(H)^{\circ} \leqslant H^{\prime} \leqslant N_{G}(H)$, then $H^{\prime}$ is $G$-cr [4, Theorem 3.14]. The relative versions of these results are as follows.

THEOREM 4.8. With the notation of Theorem 1, the following hold.

(1) Let $H^{\prime}$ be a normal subgroup of $H$. If $H$ is relatively $G$-completely reducible with respect to $K$, then so is $H^{\prime}$.

(2) Let $H^{\prime}$ be a subgroup of $N$ satisfying $(H C) N_{N}(H C)^{\circ} \leqslant H^{\prime} C \leqslant N_{N}(H C)$. If $H$ is relatively $G$-completely reducible with respect to $K$, then so is $H^{\prime}$.

(3) $H$ is relatively $G$-completely reducible with respect to $K$ if and only if $N_{N}(H C)$ is relatively $G$-completely reducible with respect to $K$.

(4) If $H$ is relatively $G$-completely reducible with respect to $K$, then so is $C_{N}(H C)$.

Proof. Part (1) follows directly from Theorem 1 and the absolute result [4, Theorem 3.10] since $\pi\left(H^{\prime}\right)$ is normal in $\pi(H)$.

For part (2), if $H$ is relatively $G$-cr with respect to $K$, then by Theorem 4.2, $\pi(H)$ is reductive. Therefore $N_{\pi(N)}(\pi(H))^{\circ}=\pi(H)^{\circ} C_{\pi(N)}(\pi(H))^{\circ}$. The group $\pi\left(N_{N}(H C)^{\circ}\right)$ is a connected subgroup of $N_{\pi(N)}(\pi(H))$ containing $\pi(H)^{\circ}$. Moreover, if $\pi(x) \cdot \pi(h)=\pi(h)$ for some $h \in H$, then $x \cdot h=h c^{\prime}$ for some $c^{\prime} \in C$. Since $C$ is normal in $N$, it follows that $x \cdot(h c) \in H C$ for all $h \in H$ and 
all $c \in C$. Hence $N_{N}(H C)^{\circ} \geqslant \pi^{-1}\left(C_{\pi(N)}(\pi(H))^{\circ}\right)$, and so $\pi\left(H C N_{N}(H C)^{\circ}\right)=$ $\pi(H) C_{\pi(N)}(\pi(H))^{\circ}$. Since we also have $\pi\left(N_{N}(H)\right) \leqslant N_{\pi(N)}(\pi(H))$, the hypotheses imply that $\pi(H) C_{\pi(N)}(\pi(H))^{\circ} \leqslant \pi\left(H^{\prime}\right) \leqslant N_{\pi(N)}(\pi(H))$. Applying the result from the absolute case [4, Theorem 3.14], we conclude that $\pi\left(H^{\prime}\right)$ is $\pi(N)$-cr, and so $H^{\prime}$ is relatively $G$-cr with respect to $K$.

For part (3), if $H$ is relatively $G$-cr with respect to $K$, then by (2) so is $N_{N}(H C)$. Conversely, if $N_{N}(H C)$ is relatively $G$-cr with respect to $K$, then by (1) so is its normal subgroup $H C$. This is contained in precisely the same parabolic and Levi subgroups corresponding to elements of $Y(K)$ as $H$, and so $H$ is also relatively $G$-cr with respect to $K$. Part (4) now follows from parts (1) and (3) since $C_{N}(H C)$ is normal in $N_{N}(H C)$.

REMARK 4.9. Theorem 4.8 fails without the hypothesis that $H \leqslant N$, even if we impose other natural conditions, for instance requiring that $H$ is connected and reductive [7, Examples 5.6 and 5.7].

The following example demonstrates the failure of Theorems 4.2(i) and 4.8(i) if $H$ does not normalize $K^{\circ}$, even when $H$ is connected.

EXAMPLE 4.10. Let $G=\mathrm{GL}_{n}(k), K=\mathrm{SO}_{n}(k)$ and let $e_{1}, \ldots, e_{n}$ be the standard basis of $k^{n}$. Suppose that $\operatorname{char}(k) \geqslant 3$ or $n \geqslant 3$. Let $H$ be the stabilizer in $G$ of the totally isotropic subspace $\left\langle e_{1}\right\rangle$. Since $H$ is relatively $G$-irreducible with respect to $K$, it is relatively $G$-completely reducible with respect to $K$. But its normal subgroup $R_{u}(H)$ is not relatively $G$-completely reducible with respect to $K$ since $R_{u}(H) \leqslant R_{u}\left(\operatorname{Stab}_{G}(f)\right)$, where $f$ is the flag $f=\left\langle e_{1}\right\rangle \leqslant\left\langle e_{1}\right\rangle^{\perp}$ and $\operatorname{Stab}_{G}(f)$ is a parabolic subgroup of $G$, which is given by a cocharacter of $K$.

In addition, $R_{u}(H)$ is not contained in $N_{G}\left(K^{\circ}\right)$. Thus $H$ does not normalize $K^{\circ}$ and $R_{u}(H)$ does not centralize $K^{\circ}$. For let $h=\left(\begin{array}{cccc}1 & 1 & \cdots & 1 \\ 0 & \ddots & 0 & \vdots \\ \vdots & 0 & \ddots & 1 \\ 0 & \cdots & 0 & 1\end{array}\right) \in R_{u}(H)$. Since $\operatorname{char}(k) \geqslant 3$ or $n \geqslant 3$, we see that $h t h^{-1} \notin K$, for $1 \neq t \in \mathrm{D}_{n} \cap K$, where $\mathrm{D}_{n}$ is the subgroup of diagonal matrices in $G$.

The following directly generalizes [5, Theorem 1.3].

COROLlaRY 4.11. With the notation of Theorem 1, suppose that $\pi(N)$ is connected and that $p>3$ or $p$ is good for $K$. Let A, B be commuting connected subgroups of $N$, which are relatively $G$-completely reducible with respect to $K$. Then $A B$ is relatively $G$-completely reducible with respect to $K$. 
Proof. This follows directly from the absolute result [5, Theorem 1.3] and Theorem 1 since $\pi(A B)$ is the commuting product of the connected groups $\pi(A)$ and $\pi(B)$.

4.3. A geometric viewpoint. We end this section with a geometric consequence of Theorem 3.

THEOREM 4.12. In the notation of Theorem 3, with $\mathbf{h} \in N^{n}$ and $\pi$ also denoting the quotient map $N^{n} \rightarrow(N / C)^{n}$, the following are equivalent.

(1) $K \cdot \mathbf{h}$ is closed in $G^{n}$.

(2) $\pi(K) \cdot \pi(\mathbf{h})$ is closed in $(N / C)^{n}$.

(3) $\pi^{-1}(\pi(K \cdot \mathbf{h}))$ is closed in $G^{n}$.

(4) Every orbit of $K$ on $\pi^{-1}(\pi(K \cdot \mathbf{h}))$ is closed in $G^{n}$.

Proof. Theorem 3 tells us that (1) and (2) are equivalent. Now $N^{n}$ is closed in $G^{n}$ and, since $\pi(K) \cdot \pi(\mathbf{h})=\pi(K \cdot \mathbf{h})$ and the topology on $(N / C)^{n}$ is the quotient topology, it follows that (2) and (3) are equivalent.

Let $K=\bigcup_{i=1}^{t} K^{\circ} x_{i}$ be the decomposition of $K$ into right cosets of $K^{\circ}$. Expressing $\mathbf{h}$ as $\mathbf{h}=\left(h_{1}, \ldots, h_{n}\right)$, we have

$$
\begin{aligned}
\pi^{-1}(\pi(K \cdot \mathbf{h})) & =\bigcup_{i=1}^{t} \pi^{-1}\left(\pi\left(K^{\circ} \cdot\left(x_{i} \cdot \mathbf{h}\right)\right)\right) \\
& =\bigcup_{i=1}^{t}\left\{\left(x \cdot\left(x_{i} \cdot h_{1}\right) c_{1}, \ldots, x \cdot\left(x_{i} \cdot h_{n}\right) c_{n}\right): x \in K^{\circ}, c_{i} \in C\right\}, \\
& =\bigcup_{i=1}^{t}\left\{\left(x \cdot\left(\left(x_{i} \cdot h_{1}\right) c_{1}\right), \ldots, x \cdot\left(\left(x_{i} \cdot h_{n}\right) c_{n}\right)\right): x \in K^{\circ}, c_{i} \in C\right\},
\end{aligned}
$$

where the last equality follows since $K^{\circ}$ centralizes $C$.

Now if $K \cdot \mathbf{h}$ is closed in $G^{n}$, then so is $K^{\circ} \cdot\left(x_{i} \cdot \mathbf{h}\right)$ for each $i$. Thus $\pi^{-1}(\pi(K \cdot \mathbf{h}))$ is a union of $K^{\circ}$-orbits, each of which is translated to one of the closed $K^{\circ}$-orbits $K^{\circ} \cdot\left(x_{i} \cdot \mathbf{h}\right)$ by an element of $C^{n}$. As translation is a variety automorphism $G^{n} \rightarrow G^{n}$, it follows that every $K^{\circ}$-orbit in $\pi^{-1}(\pi(K \cdot \mathbf{h}))$ is closed in $G^{n}$. Consequently, every $K$-orbit in $\pi^{-1}(\pi(K \cdot \mathbf{h}))$ is closed in $G^{n}$ as well. So (4) follows from (1), and the reverse implication is clear.

REMARK 4.13. The proofs of the equivalences (2) $\Leftrightarrow$ (3) and (1) $\Leftrightarrow$ (4) in Theorem 4.12 are easily seen to be independent of Theorems 1 and 3. From the 
argument above, we see that $\pi^{-1}(\pi(K \cdot \mathbf{h}))$ consists of $K^{\circ}$-orbits, which are $C^{n}$ translates of a $K^{\circ}$-orbit $K^{\circ} \cdot\left(x_{i} \cdot \mathbf{h}\right)$ for some $i$. From the observation that one of the latter is closed if and only if all of them are closed, together with the fact that closed orbits always exist, we conclude that all $K^{\circ}$-orbits are closed in $\pi^{-1}(\pi(K \cdot \mathbf{h}))$ in the subspace topology and thus every $K$-orbit in $\pi^{-1}(\pi(K \cdot \mathbf{h}))$ is closed in $\pi^{-1}(\pi(K \cdot \mathbf{h}))$ in the subspace topology. Hence we also see that (3) implies (4), even without appealing to Theorem 3 (or Theorem 1). However, the final implication is more subtle since it is not clear a priori that in this setting, an arbitrary union of $C^{n}$-translates of a closed $K$-orbit is again Zariski-closed in $G^{n}$.

Corollary 3.9 also has a geometric counterpart, as follows. If $\mathbf{h} \in G^{n}$ is a generic tuple for a subgroup $H \leqslant G$, then $H$ is relatively $G$-irreducible with respect to $K$ precisely when $\mathbf{h}$ is a $K$-stable point of $G^{n}$, that is, $K \cdot \mathbf{h}$ is closed in $G^{n}$ and $C_{K}(\mathbf{h}) / C_{K}(G)$ is finite [7, Definition 3.12, Proposition 3.16].

COROLLARY 4.14. In the notation of Theorem 3, suppose that $K^{\circ}$ is semisimple and that $\mathbf{h} \in N^{n}$. Then the following are equivalent.

(1) $\mathbf{h}$ is a $K$-stable point of $G^{n}$.

(2) $\pi(\mathbf{h})$ is a $\pi(K)$-stable point of $(N / C)^{n}$.

(3) $\pi^{-1}(\pi(K \cdot \mathbf{h}))$ is closed in $G^{n}$ and contains a $K$-stable point.

(4) Every point of $\pi^{-1}(\pi(K \cdot \mathbf{h}))$ is $K$-stable in $G^{n}$.

Proof. Corollary 3.9 gives the equivalence $(1) \Leftrightarrow(2)$. Theorem 4.12 tells us that the closure conditions in (1), (3) and (4) are equivalent. It therefore suffices to show that all $K$-orbits in $\pi^{-1}(\pi(K \cdot \mathbf{h}))$ have the same dimension, since then their stabilizers (in $K$ ) all have the same dimension, so $\pi^{-1}(\pi(K \cdot \mathbf{h}))$ contains a $K$-stable point if and only if all of its points are $K$-stable. As in the proof of Theorem 4.12, the set $\pi^{-1}(\pi(K \cdot \mathbf{h}))$ is a union of $K^{\circ}$-orbits, each of which is a $C^{n}$-translate of a $K^{\circ}$-orbit $K^{\circ} x_{i} \cdot \mathbf{h}$, where $\left\{x_{1}, \ldots, x_{t}\right\}$ is a set of coset representatives for $K^{\circ}$ in $K$. But $K / K^{\circ}$ acts transitively by conjugation on these orbits since $K^{\circ} x_{i} \cdot \mathbf{h}=x_{i} K^{\circ} \cdot \mathbf{h}=x_{i} \cdot\left(K^{\circ} \cdot \mathbf{h}\right)$; hence these orbits indeed have the same dimension.

\section{Relative complete reducibility and separability}

Recall from [4, Definition 3.27] that a closed subgroup $H$ of $G$ is called separable in $G$ if the Lie algebra centralizer $C_{\mathrm{Lie}(G)}(H)$ equals the Lie algebra of $C_{G}(H)$. As discussed in [4, Section 3.5], this is equivalent to the smoothness 
of the scheme-theoretic centralizer of $H$ in $G$. Moreover, if $H$ is topologically generated by $\left\{h_{1}, \ldots, h_{n}\right\}$, then $H$ is separable in $G$ precisely when the orbit map $G \rightarrow G \cdot\left(h_{1}, \ldots, h_{n}\right)$ is a separable morphism of varieties.

In [4, Section 3.5] and [6], it is shown that separability interacts closely with complete reducibility. We now derive relative analogues of these results.

DEFINITION 2. For subgroups $H$ and $K$ of a reductive group $G$, we say that $H$ is separable for $K$ if $\operatorname{Lie}\left(C_{K}(H)\right)$ and $C_{\operatorname{Lie}(K)}(H)$ coincide as subspaces of $\operatorname{Lie}(G)$.

This definition is equivalent to requiring that the orbit map $K \rightarrow K \cdot \mathbf{h}$ is a separable morphism for some (equivalently any) generic tuple $\mathbf{h}$ of $H$. This equivalence is proved in [2, Lemma 5.1] under the assumption that $H \leqslant K$ and $K$ is connected, but the same proof applies word for word in this more general setting. Note also that when $K=G$, this gives the usual definition of separability of a subgroup in $G$; cf. [4, Definition 3.27].

Recall from [7, Theorem 3.5] that a subgroup of $G$ is relatively $G$-cr with respect to $K$ if and only if for all $\lambda \in Y(K)$ such that $H \leqslant P_{\lambda}$, we have $\operatorname{dim} C_{K}(H)=\operatorname{dim} C_{K}\left(c_{\lambda}(H)\right)$, where $c_{\lambda}$ is the map $P_{\lambda} \rightarrow L_{\lambda}$, given by $x \mapsto$ $\lim _{a \rightarrow 0}(\lambda(a) \cdot x)$. This observation allows us to prove the following analogue of [4, Theorem 3.46].

THEOREM 5.1. In the notation of Theorem 1, suppose that $H$ is separable for $K$. If $\operatorname{Lie}(K)$ is semisimple as an $H$-module, then $H$ is relatively $G$-completely reducible with respect to $K$.

Proof. We mimic the proof of [4, Theorem 3.46]. Suppose that $H$ is not relatively $G$-cr with respect to $K$. Thus by [7, Theorem 3.5], there exists $\lambda \in Y(K)$ such that $H \leqslant P_{\lambda}$ and $\operatorname{dim} C_{K}(H)<\operatorname{dim} C_{K}\left(c_{\lambda}(H)\right)$. Since $H$ is separable for $K$ by hypothesis, it follows that

$$
\operatorname{dim} C_{\operatorname{Lie}(K)}(H)=\operatorname{dim} C_{K}(H)<\operatorname{dim} C_{K}\left(c_{\lambda}(H)\right) \leqslant \operatorname{dim} C_{\operatorname{Lie}(K)}\left(c_{\lambda}(H)\right) .
$$

Note that $c_{\lambda}(H)$ is in $N$ : for each $a$ in $k$, and $x$ in $N$, we have that $\lambda(a) x \lambda(a)^{-1}$ belongs to $N$. As $N$ is closed, the limit of the former as $a$ tends to 0 still belongs to $N$. In particular, $c_{\lambda}(H)$ belongs to $N$.

Now consider the images of $H$ and $c_{\lambda}(H)$ under the adjoint map Ad: $N \rightarrow$ $\mathrm{GL}(\operatorname{Lie}(K))$. Then it is clear that $\operatorname{Ad}\left(c_{\lambda}(H)\right)=c_{\operatorname{Ad} \circ \lambda}(H)$. Since $\operatorname{Lie}(K)$ is semisimple as an $H$-module, we deduce that $\operatorname{Ad}(H)$ is $\mathrm{GL}(\operatorname{Lie}(K))$-completely reducible, and in particular it is $\operatorname{GL}(\operatorname{Lie}(K))$-conjugate to $\operatorname{Ad}\left(c_{\lambda}(H)\right)$. The fixed points of $\operatorname{Ad}(H)$ and $\operatorname{Ad}\left(c_{\lambda}(H)\right)$ on $\operatorname{Lie}(K)$ are precisely $C_{\operatorname{Lie}(K)}(H)$ and 
$C_{\mathrm{Lie}(K)}\left(c_{\lambda}(H)\right)$, and so these have equal dimensions. This contradicts the strict inequality above.

REMARK 5.2. One may be tempted to prove Theorem 5.1 more directly by working with the image in $\pi(N)$ and applying Theorem 1 and the absolute result [4, Theorem 3.46]. However, even in the absolute case, separability of $H$ does not imply separability of $\pi(H)$ in $\pi(N)$. As an example of this phenomenon, it is well known that every subgroup of $\mathrm{GL}_{n}(k)$ is separable, whereas $\mathrm{PGL}_{n}(k)$ has nonseparable subgroups, for instance the normalizer of a maximal torus in $\mathrm{PGL}_{2}(k)$ is not separable when $\operatorname{char}(k)=2$; cf. [4, Examples 3.28-3.30].

REMARK 5.3. In [6, Theorem 1.2], it is shown that if $G$ is connected and char $(k)$ is very good for $G$, then every subgroup of $G$ is separable in $G$. This does not generalize to nonconnected $G$. Again, the normalizer of a maximal torus in $\operatorname{PGL}_{2}(k)$ provides a counterexample when $\operatorname{char}(k)=2$. This subgroup has the form $G=T \rtimes\langle x\rangle$, where $x$ is an involution inverting every element of the 1dimensional torus $T$. Then $G$ is centreless but acts trivially on the 1-dimensional Lie algebra $\operatorname{Lie}(G)$; in particular, $G$ is not separable as a subgroup of itself, although 2 is very good for $G$ as the root system is trivial (cf. [6, Remark 3.5(iv)], which is missing the necessary condition $p=2$ ).

It turns out that the above is essentially the only obstruction, arising because $\operatorname{char}(k)$ divides the order of the finite group $T \rtimes\langle x\rangle / T$. The following generalizes [6, Theorem 1.2] both to nonconnected $G$ and to the relative setting.

THEOREM 5.4. With the notation of Theorem 1, suppose that char $(k)$ is zero or is very good for $K$ and coprime to $\left|\pi(H) / \pi\left(H \cap N^{\circ}\right)\right|$. Then $H$ is separable for $K$. In particular, if char $(k)$ is zero or is very good for $K$ and coprime to one of $\left|\pi(H) / \pi\left(H^{\circ}\right)\right|$ or $\left|\pi(N) / \pi\left(N^{\circ}\right)\right|$, then $H$ is separable for $K$.

Proof. For brevity, write $H^{\prime}=H \cap N^{\circ}$. Note first that $H / H^{\prime} \cong H N^{\circ} / N^{\circ} \leqslant$ $N / N^{\circ}$, and since $H^{\circ} \leqslant H^{\prime}$, we have a surjection $H / H^{\circ} \rightarrow H / H^{\prime}$. Applying $\pi$, if $\operatorname{char}(k)$ is positive and coprime to one of $\left|\pi(H) / \pi\left(H^{\circ}\right)\right|$ or $\left|\pi(N) / \pi\left(N^{\circ}\right)\right|$, then it is coprime to $\left|\pi(H) / \pi\left(H^{\prime}\right)\right|$. Thus it suffices to prove the first statement of the theorem.

Now, $C_{K}(H)^{\circ}$ is the largest connected subgroup of $K$ centralizing $H$ and hence is the largest connected subgroup of $K^{\circ}$ centralizing $H$; thus $\operatorname{dim} C_{K}(H)=$ $\operatorname{dim} C_{K^{\circ}}(H)$. Since also $\operatorname{Lie}(K)=\operatorname{Lie}\left(K^{\circ}\right)$, it follows that we can assume $K=K^{\circ}$. Next let $M$ be a reductive subgroup of $N$ guaranteed by Lemma 3.1. It is clear that $\operatorname{dim} C_{K}(H)=\operatorname{dim} C_{K}(H C)$ and $C_{\mathrm{Lie}(K)}(H)=C_{\mathrm{Lie}(K)}(H C)$, and 
since $H C=(H C \cap M) C$, it suffices to prove the result assuming that $H \leqslant M$ (this does not change $\pi(H)$ or $\pi\left(H \cap N^{\circ}\right)$ ). We can therefore also assume $G=N=M Z(K)^{\circ}$, since this does not change $K, C_{K}(H), C_{\operatorname{Lie}(K)}(H), \pi(H)$ or $\pi\left(H \cap N^{\circ}\right)$. In this case, $H$ is separable for $K$ precisely when $H$ is separable in $G$.

We now have $G^{\circ}=N^{\circ}=K$; hence $\operatorname{char}(k)$ is very good for $G$ by hypothesis. Now $H^{\prime}=H \cap N^{\circ}=H \cap G^{\circ}$; in particular, $H^{\prime} \leqslant G^{\circ}$ and by [6, Theorem 1.2], the subgroup $H^{\prime}$ is separable in $G^{\circ}$. The finite group $H / H^{\prime}$ acts on $C_{K}\left(H^{\prime}\right)$ and on $C_{\operatorname{Lie}(K)}\left(H^{\prime}\right)$, and $H$ is separable in $G$ (hence separable for $K$ ) precisely when the fixed points of $H / H^{\prime}$ on $\operatorname{Lie} C_{K}\left(H^{\prime}\right)=C_{\operatorname{Lie}(K)}\left(H^{\prime}\right)$ are equal to the Lie algebra of $C_{C_{K}\left(H^{\prime}\right)}\left(H / H^{\prime}\right)$. But the action of $H$ on $K$ factors through $\pi(H)$, and so the action of $H / H^{\prime}$ factors through $\pi(H) / \pi\left(H^{\prime}\right)$. By hypothesis, $\operatorname{char}(k)$ is either zero or coprime to the order of this latter group; hence $\pi(H) / \pi\left(H^{\prime}\right)$ is linearly reductive and the desired result follows from [16, Lemma 4.1].

Combining Theorems 5.4 and 5.1 gives the following, which in turn implies Theorem 4.

COROLLARY 5.5. With the notation of Theorem 1 , suppose that $\operatorname{char}(k)$ is zero or is very good for $K$ and coprime to $\left|\pi(H) / \pi\left(H \cap N^{\circ}\right)\right|$. If Lie $(K)$ is semisimple as an $H$-module, then $H$ is relatively $G$-completely reducible with respect to $K$.

In [4, Section 3.5] and [6], it is shown that separability interacts closely with Richardson's notion of reductive pairs. We now generalize these results to the relative setting.

Definition 3. Let $G$ be reductive, let $H, K \leqslant G$ and suppose that $H$ normalizes $K^{\circ}$. We say that $(G, K)$ is a reductive pair for $H$ if $\operatorname{Lie}(K)$ is an $H$-module direct summand of $\operatorname{Lie}(G)$.

This generalizes the usual notion of a reductive pair [4, Definition 3.32], which is the special case $H=K$.

The following result generalizes [2, Corollary 5.3], which is a corollary of [2, Lemma 5.2]. The proof of this latter result does not use the assumption ' $H \leqslant K$ ', and therefore goes through word for word in this situation.

LEMMA 5.6 (Cf. [2, Corollary 5.3]). Let $K \leqslant G$ be reductive algebraic groups and let $H$ be a subgroup of $G$. If $H$ is separable for $G$ and $(G, K)$ is a reductive pair for $H$, then $H$ is separable for $K$.

The following corollary of Lemma 5.6 generalizes [2, Corollary 5.4] to the case that $G$ is not necessarily connected. 
Corollary 5.7. Take $G, K, H$ as in Lemma 5.6 and let $\psi: G \rightarrow \operatorname{Aut}\left(G^{\circ}\right)$ denote the map induced by conjugation. If $(G, K)$ is a reductive pair for $H$ and $\operatorname{char}(k)$ is zero, or is very good for $G$ and coprime to $\left|\psi(H) / \psi\left(H \cap G^{\circ}\right)\right|$, then $H$ is separable for $K$.

Proof. Apply Theorem 5.4 (taking $K=G$ there) to conclude that $H$ is separable for $G$, and then apply Lemma 5.6.

Proof of Theorem 5. It is well known that every subgroup of GL $(V)$ is separable in $\mathrm{GL}(V)$; hence Lemma 5.6 implies that $H$ is separable for $K$. Again, since $\operatorname{Lie}(K)$ is a semisimple $H$-module, the desired conclusion follows from Theorem 5.1.

Combining Corollary 5.7 and Theorem 5.1 gives the following, which in turn implies Theorem 6 .

COROLlaRY 5.8. Let $K \leqslant G$ be reductive algebraic groups, let $H$ be a subgroup of $G$ and let $\psi: G \rightarrow \operatorname{Aut}\left(G^{\circ}\right)$ denote the map induced by conjugation. Suppose that $(G, K)$ is a reductive pair for $H$ and $c h a r(k)$ is either zero or is very good for $G$ and coprime to $\left|\psi(H) / \psi\left(H \cap G^{\circ}\right)\right|$. If $\mathrm{Lie}(K)$ is semisimple as an $H$-module, then $H$ is relatively $G$-completely reducible with respect to $K$.

REMARK 5.9. Theorem 5.4, Corollary 5.7 and Corollary 5.8 all hold with the term $\left|H /\left(H \cap N^{\circ}\right)\right|$ in place of $\left|\pi(H) / \pi\left(H \cap N^{\circ}\right)\right|$ and other similar adjustments. While this makes for slightly cleaner statements, it also misses some pathological cases, such as letting $K$ be a connected reductive group and $G=H=K \times S$ for a finite $p$-group $S$, where $p=\operatorname{char}(k)>0$. In this case, the question of whether $H$ is separable for $K$ does not depend on properties of $S$, although $H /(H \cap K) \cong S$ so $\operatorname{char}(k)$ divides $\left|H /\left(H \cap G^{\circ}\right)\right|$ if $|S|>0$.

\section{Relative complete reducibility of Lie subalgebras}

In this section, we consider the analogue of Theorem 1 for subalgebras of $\operatorname{Lie}(G)$. We write $\mathfrak{g}=\operatorname{Lie}(G)$, and we denote the Lie algebras of the subgroups $K, N, C, P_{\lambda}, L_{\lambda}$ of $G$ by $\mathfrak{k}, \mathfrak{n}, \mathfrak{c}, \mathfrak{p}_{\lambda}$ and $\mathfrak{l}_{\lambda}$, respectively.

Recall from [7, Definition 3.9] that a subalgebra $\mathfrak{h}$ of $\mathfrak{g}$ is called relatively $G$ completely reducible with respect to a reductive subgroup $K \leqslant G$ if, whenever $\mathfrak{h} \subseteq \mathfrak{p}_{\lambda}$ for $\lambda \in Y(K)$, there exists $\mu \in Y(K)$ such that $P_{\lambda}=P_{\mu}$ and $\mathfrak{h} \subseteq \mathfrak{l}_{\mu}$. If this holds for $K=G$, then $\mathfrak{h}$ is called $G$-completely reducible; cf. [15].

LEMMA 6.1. In the notation of Theorem 1 , for all $\lambda \in Y(K)$, the following hold.

(1) $\pi\left(P_{\lambda}^{\circ} \cap N\right)=P_{\pi \circ \lambda}^{\circ}, \pi\left(L_{\lambda}^{\circ} \cap N\right)=L_{\pi \circ \lambda}^{\circ}$. 
(2) $\pi^{-1}\left(P_{\pi \circ \lambda}^{\circ}\right)=P_{\lambda}^{\circ} \cap N, \pi^{-1}\left(L_{\pi \circ \lambda}^{\circ}\right)=L_{\lambda}^{\circ} \cap N$.

(3) $\operatorname{Lie}\left(P_{\lambda} \cap N\right)=\mathfrak{p}_{\lambda} \cap \mathfrak{n}, \operatorname{Lie}\left(L_{\lambda} \cap N\right)=\mathfrak{l}_{\lambda} \cap \mathfrak{n}$.

(4) $d \pi\left(\mathfrak{p}_{\lambda} \cap \mathfrak{n}\right)=\mathfrak{p}_{\pi \circ \lambda}, d \pi\left(\mathfrak{l}_{\lambda} \cap \mathfrak{n}\right)=\mathfrak{l}_{\pi \circ \lambda}$.

(5) $(d \pi)^{-1}\left(\mathfrak{p}_{\pi \circ \lambda}\right)=\mathfrak{p}_{\lambda} \cap \mathfrak{n},(d \pi)^{-1}\left(\mathfrak{l}_{\pi \circ \lambda}\right)=\mathfrak{l}_{\lambda} \cap \mathfrak{n}$.

Proof. For all $\lambda \in Y(K)$, we have $P_{\pi \circ \lambda}^{\circ}=P_{\pi \circ \lambda} \cap(N / C)^{\circ}$ since this is a parabolic subgroup of the connected reductive subgroup $(N / C)^{\circ}$, and similarly $L_{\pi \circ \lambda}^{\circ}=L_{\pi \circ \lambda} \cap(N / C)^{\circ}$. Now recall the subgroup $M$ from Lemma 3.1, and that the restriction $\pi: M \rightarrow N / C$ is an isogeny. Thus $\pi: M^{\circ} \rightarrow(N / C)^{\circ}$ is a surjective map of connected reductive groups. Thus part (1) follows from [4, Lemma 2.11(i)]. For part (2), using part (1), we see that $\pi^{-1}\left(P_{\pi \circ \lambda}^{\circ}\right)=\pi^{-1}\left(\pi\left(P_{\lambda}^{\circ}\right)\right)=\left(P_{\lambda}^{\circ} \cap N\right) C=P_{\lambda}^{\circ} \cap N$, and similarly for $L_{\lambda}$.

Part (3) follows from the proof of [19, Theorem 13.4.2(ii)], which shows that the containment (63) given on p. 234 there is an equality; this is the desired result for $P_{\lambda}$. Also, $\operatorname{Lie}\left(L_{\lambda} \cap N\right)$ is the subalgebra of $\mathfrak{n}$ centralized by $\lambda\left(k^{*}\right)$, which is precisely $\mathfrak{l}_{\lambda} \cap \mathfrak{n}$.

For part (4), the left-hand side is clearly contained on the right-hand side. On the other hand, $\mathfrak{n}=\operatorname{Lie}\left(N^{\circ}\right)$ and the restriction $\pi: N^{\circ} \rightarrow N^{\circ} /\left(N^{\circ} \cap C\right)$ is a quotient of $N^{\circ}$ by the closed subgroup $N^{\circ} \cap C$ and is therefore separable [19, 5.5.6(ii)] (cf. also [19, Exercise 5.5.9(5)(c)]); so $d \pi$ induces an isomorphism $\mathfrak{n} / \mathfrak{c} \rightarrow \operatorname{Lie}(N / C)$. Therefore,

$$
\begin{aligned}
\operatorname{dim} d \pi\left(\mathfrak{p}_{\lambda} \cap \mathfrak{n}\right)+\operatorname{dim} \mathfrak{c} & =\operatorname{dim}\left(\mathfrak{p}_{\lambda} \cap \mathfrak{n}\right) \\
& =\operatorname{dim}\left(P_{\lambda}^{\circ} \cap N\right) \\
& =\operatorname{dim} \pi\left(P_{\lambda}^{\circ} \cap N\right)+\operatorname{dim} C \\
& =\operatorname{dim} P_{\pi \circ \lambda}+\operatorname{dim} C \\
& =\operatorname{dim} \mathfrak{p}_{\pi \circ \lambda}+\operatorname{dim} C,
\end{aligned}
$$

where the first equality uses the separability of the restriction of $\pi$ to $N^{\circ}$ and the second equality uses (3). We thus deduce that $\operatorname{dim} d \pi\left(\mathfrak{p}_{\lambda} \cap \mathfrak{n}\right)=\operatorname{dim} \mathfrak{p}_{\pi \circ \lambda}$, and so the two subalgebras are equal. An identical argument shows that $d \pi\left(\mathfrak{l}_{\lambda} \cap \mathfrak{n}\right)=\mathfrak{l}_{\pi \circ \lambda}$.

Part (5) follows directly from part (4), as $(d \pi)^{-1}\left(\mathfrak{p}_{\pi \circ \lambda}\right)=(d \pi)^{-1}\left(d \pi\left(\mathfrak{p}_{\lambda} \cap \mathfrak{n}\right)\right)=$ $\mathfrak{p}_{\lambda} \cap \mathfrak{n}$, and similarly for $\mathfrak{l}_{\lambda}$.

Our analogue of Theorem 1 for Lie algebras is now as follows.

THEOREM 6.2. Let $K \leqslant G$ be reductive algebraic groups. Let $N=N_{G}\left(K^{\circ}\right) \leqslant$ $N_{G}(\mathfrak{k})$ and $C=C_{G}\left(K^{\circ}\right) \leqslant C_{G}(\mathfrak{k})$, let $\pi: N \rightarrow N / C$ be the quotient map, and write $d \pi$ for the differential $\mathfrak{n} \rightarrow \mathfrak{n} / \mathfrak{c}=\operatorname{Lie}(N / C)$. 
Let $\mathfrak{h}$ be a Lie subalgebra of $\mathfrak{n}$. Then $\mathfrak{h}$ is relatively $G$-completely reducible with respect to $K$ if and only if $d \pi(\mathfrak{h})$ is $\pi(N)$-completely reducible.

Proof. The map $\pi: N \rightarrow N / C$ induces a surjection $Y(K) \rightarrow Y(N / C)$. It follows from Lemma 6.1(iv), (v) that $\mathfrak{h}$ is contained in $\mathfrak{p}_{\lambda}$ (respectively, $\mathfrak{l}_{\lambda}$ ) for $\lambda \in Y(K)$ if and only if $d \pi(\mathfrak{h})$ is contained in $\mathfrak{p}_{\pi \circ \lambda}$ (respectively, $\mathfrak{l}_{\pi \circ \lambda}$ ).

The following is the Lie algebra counterpart of Corollary 4.1. Since both $\mathfrak{n} / \mathfrak{c}$ and the trivial subalgebra in $\mathfrak{n} / \mathfrak{c}$ are $\pi(N)$-cr, Theorem 6.2 gives the following.

COROLlary 6.3. Let $K \leqslant G$ be reductive algebraic groups. Then the subalgebras $\mathfrak{n}_{\mathfrak{g}}\left(K^{\circ}\right)$ and $\mathfrak{c}_{\mathfrak{g}}\left(K^{\circ}\right)$ of $\mathfrak{g}$ are relatively $G$-completely reducible with respect to $K$.

As with relative $G$-complete reducibility of subgroups, a Lie subalgebra $\mathfrak{h}$ of $\mathfrak{g}$ is relatively $G$-cr with respect to a reductive subgroup $K \leqslant G$ precisely when the $K$-orbit of any (equivalently, of every) finite tuple $\mathbf{h} \in \mathfrak{h}^{n}$ which generates $\mathfrak{h}$ as a Lie algebra is closed in $\mathfrak{g}^{n}$ [7, Theorem 3.10(iii)]. Thus results from geometric invariant theory can be brought to bear. In particular, if $\mathfrak{h}$ is not relatively $G$-cr with respect to $K$, then there exists a so-called 'optimal destabilizing parabolic subgroup' for $\mathfrak{h}$; see [7, Definition 3.23 and Remark 3.24]. This is a canonical parabolic subgroup $P_{\lambda}(\lambda \in Y(K))$ such that $\mathfrak{h} \subseteq \mathfrak{p}_{\lambda}$ and $\mathfrak{h} \nsubseteq \mathfrak{l}_{\lambda}$.

The following result generalizes [15, Theorem 1(2)]. It follows directly from this absolute result and Theorem 6.2, but can also be proved directly by considering optimal destabilizing parabolic subgroups, mirroring [8, Example 5.29].

THEOREM 6.4. In the notation of Theorem 1 , if $H$ is relatively $G$-completely reducible with respect to $K$, then the Lie algebra $\mathfrak{h}$ of $H$ is also relatively $G$ completely reducible with respect to $K$.

Proof. Suppose that $\mathfrak{h}$ is not relatively $G$-cr with respect to $K$ and let $P_{\lambda}$ ( $\lambda \in$ $Y(K))$ be the optimal destabilizing parabolic subgroup for $\mathfrak{h}$. By the optimality of $P_{\lambda}$ [7, Remark 3.24], we have $N_{N_{G}(\mathfrak{k})}(\mathfrak{h}) \leqslant P_{\lambda}$, and since $H \leqslant N_{G}(\mathfrak{h})$ and $H \leqslant N \leqslant N_{G}(\mathfrak{k})$, we have $H \leqslant P_{\lambda}$ also. Then by hypothesis, we have $H \leqslant L_{\mu}$ for some $\mu \in Y(K)$ such that $P_{\lambda}=P_{\mu}$, and therefore $\mathfrak{h} \leqslant \mathfrak{l}_{\mu}$.

Note that the converse of Theorem 6.4 already fails in the absolute case [15].

Here is the counterpart of Theorem 3 for Lie algebras, which is equivalent to Theorem 6.2 thanks to [7, Theorem 3.10(iii)], which is the analogue of Theorem 2.1 for Lie subalgebras of $\mathfrak{g}$ and their generating tuples. 
THEOREM 6.5. Let $K \leqslant G$ be reductive algebraic groups, write $N=N_{G}\left(K^{\circ}\right)$, $C=C_{G}\left(K^{\circ}\right)$, and let $\pi: N \rightarrow N / C$ be the quotient map, and write $d \pi$ for the differential $\mathfrak{n} \rightarrow \mathfrak{n} / \mathfrak{c}=\operatorname{Lie}(N / C)$. Let $\mathbf{h} \in \mathfrak{n}^{n}(n \geqslant 1)$ and write $d \pi$ also for the map $\mathfrak{n}^{n} \rightarrow(\mathfrak{n} / \mathfrak{c})^{n}$.

Then $K \cdot \mathbf{h}$ is closed in $\mathfrak{g}^{n}$ if and only if $\pi(N) \cdot d \pi(\mathbf{h})$ is closed in $(\mathfrak{n} / \mathfrak{c})^{n}$.

The following is the analogue of Theorem 4.12 for the diagonal action of $K$ on $\mathfrak{g}^{n}$.

COROLLARY 6.6. With the above notation, the following are equivalent.

(1) $K \cdot \mathbf{h}$ is closed in $\mathfrak{g}^{n}$.

(2) $\pi(K) \cdot d \pi(\mathbf{h})$ is closed in $(\mathfrak{n} / \mathfrak{c})^{n}$.

(3) $(d \pi)^{-1}(d \pi(K \cdot \mathbf{h}))$ is closed in $\mathfrak{g}^{n}$.

(4) Every orbit of $K$ on $(d \pi)^{-1}(d \pi(K \cdot \mathbf{h}))$ is closed in $\mathfrak{g}^{n}$.

Proof. The equivalence of (1) and (2) is given by Theorem 6.5. The equivalence of (2) and (3) follows from the fact that the topology on $(\mathfrak{n} / \mathfrak{c})^{n}$ is the quotient topology, and we also use the fact that $d \pi$ is surjective (as shown in the proof of Lemma 6.1(4)).

Let $K=\bigcup_{i=1}^{t} K^{\circ} x_{i}$ be the decomposition of $K$ into right cosets of $K^{\circ}$. Writing $\mathbf{h}=\left(h_{1}, \ldots, h_{n}\right)$, we have

$$
\begin{aligned}
(d \pi)^{-1}(d & \pi(K \cdot \mathbf{h}))=\bigcup_{i=1}^{t}(d \pi)^{-1}\left(d \pi\left(K^{\circ} \cdot\left(x_{i} \cdot \mathbf{h}\right)\right)\right) \\
& =\bigcup_{i=1}^{t}\left\{\left(x \cdot\left(x_{i} \cdot h_{1}\right)+c_{1}, \ldots, x \cdot\left(x_{i} \cdot h_{n}\right)+c_{n}\right): x \in K^{\circ}, c_{i} \in \mathfrak{c}\right\}, \\
& =\bigcup_{i=1}^{t}\left\{\left(x \cdot\left(\left(x_{i} \cdot h_{1}\right)+c_{1}\right), \ldots, x \cdot\left(\left(x_{i} \cdot h_{n}\right)+c_{n}\right)\right): x \in K^{\circ}, c_{i} \in \mathfrak{c}\right\},
\end{aligned}
$$

where the last equality follows since $K^{\circ}$ centralizes $\mathfrak{c}$.

Now if $K \cdot \mathbf{h}$ is closed in $\mathfrak{g}^{n}$, then so is $K^{\circ} \cdot\left(x_{i} \cdot \mathbf{h}\right)$ for each $i$. Thus $(d \pi)^{-1}(d \pi(K \cdot \mathbf{h}))$ is a union of $K^{\circ}$-orbits, each of which is translated to one of the closed $K^{\circ}$-orbits $K^{\circ} \cdot\left(x_{i} \cdot \mathbf{h}\right)$ by an element of $\mathfrak{c}^{n}$. As translation is a variety automorphism $\mathfrak{g}^{n} \rightarrow \mathfrak{g}^{n}$, it follows that every $K^{\circ}$-orbit in $(d \pi)^{-1}(d \pi(K \cdot \mathbf{h}))$ is closed in $\mathfrak{g}^{n}$. Consequently, every $K$-orbit in $(d \pi)^{-1}(d \pi(K \cdot \mathbf{h}))$ is closed in $\mathfrak{g}^{n}$ as well. So (4) follows from (1) and the reverse implication is clear. 
Comments concerning the equivalences in Corollary 6.6 similar to those in Remark 4.13 apply. Moreover, one defines relative $G$-irreducibility of Lie subalgebras of $\mathfrak{g}$ (with respect to $K$ ) in terms of $K$-stable points on $\mathfrak{g}^{n}$ [7, Definition 3.12], and results analogous to Corollary 3.9 and 4.14 apply in this case, with the obvious modifications.

\section{Changing the field}

In this section, we prove Theorem 7.4, which generalizes Theorem 1 to arbitrary fields. This allows us to generalize many other results, including Theorem 3. First, we recall some relevant notions from [7, Section 4]. In this section, $k$ denotes an arbitrary field and $\bar{k}$ is the algebraic closure of $k$. Algebraic groups and varieties are taken to be defined over $\bar{k}$. If $V$ is a $k$-variety and $k^{\prime} / k$ is an algebraic extension, then we denote the set of $k^{\prime}$-points of $V$ by $V\left(k^{\prime}\right)$. The set of $k$-defined cocharacters of a $k$-group $M$ is denoted by $Y_{k}(M)$. We say that a $G$-variety $V$ is defined over $k$ if both $V$ and the action of $G$ on $V$ are defined over $k$.

We begin with the definition of relative $G$-complete reducibility over $k$, [7, Definition 4.1].

Definition 4 . Let $K \leqslant G$ be reductive algebraic $k$-groups. A subgroup $H$ of $G$ is relatively $G$-completely reducible over $k$ with respect to $K$ if for every $\lambda \in$ $Y(K)$ such that $P_{\lambda}$ is $k$-defined and $H \subseteq P_{\lambda}$, there exists $\mu \in Y(K)$ such that $P_{\lambda}=P_{\mu}, L_{\mu}$ is $k$-defined and $H \subseteq L_{\mu}$. If $K=G$, then we say that $H$ is $G$ completely reducible over $k$; see also [18] and [4, Section 5].

When $k=\bar{k}$, this definition coincides with Definition 1 since in this case each R-parabolic subgroup and R-Levi subgroup of $G$ is $k$-defined.

According to [7, Lemma 4.8], when discussing relative $G$-complete reducibility over $k$ with respect to $K$, it suffices to consider R-parabolic subgroups and RLevi subgroups of the form $P_{\lambda}$ and $L_{\lambda}$ for $\lambda \in Y_{k}(K)$, rather than all $k$-defined R-parabolic subgroups and R-Levi subgroups arising from a cocharacter of $K$.

7.1. Rational analogue of Theorem 1. We now embark on proving the rational version of Theorem 1. We need to generalize results from Section 3 and from [4]. From now on, we suppose that in the setting of Theorem 1, the groups $G$, $K, N$ and $C$ are all $k$-defined. Then the quotient map $\pi: N \rightarrow N / C$ is a $k$-defined morphism. Let $M$ be a reductive subgroup of $G$ guaranteed by Lemma 3.1, and let $\pi_{M}$ denote the restriction of $\pi$ to $M$, so that $\pi_{M}$ is an isogeny $M \rightarrow N / C$.

LEMMA 7.1. With the above assumptions, the subgroup $M$ may be taken to be $k$-defined, and then $\pi_{M}$ is a $k$-defined isogeny. 
Proof. By assumption, $K$ is $k$-defined, and hence so are $K^{\circ}$ and $\left[K^{\circ}, K^{\circ}\right]$ [9, Ch. I 1.2 Proposition (b); 2.3 Corollary]. Therefore, so is the quotient map $N \rightarrow N /\left[K^{\circ}, K^{\circ}\right]$. Now [10, Theorem 1.1] applies to the more general setting of $k$-group schemes with finite quotients; hence the subgroup $M$ constructed in Lemma 3.1 is the preimage (under $\pi$ ) of a finite $k$-defined subgroup of $N / C$ and hence is a $k$-defined subgroup of $G$. Now $\pi$ is $k$-defined and hence so is its restriction to $M$.

LEMMA 7.2. Keeping the above assumptions, suppose that $C$ is normal in $G$. If $\lambda \in Y_{k}(G / C)$, then there exists $\mu \in Y_{k}(G / C)$ such that $P_{\mu}=P_{\lambda}, L_{\mu}=L_{\lambda}$ and $\mu=\pi \circ v$ for some $v \in Y_{k}(M)$.

Proof. With $M$ and $\pi_{M}$ as above, $\pi_{M}$ is an isogeny and hence $\pi_{M}$ is quasicentral. Since $d \pi: \mathfrak{n} \rightarrow \mathfrak{n} / \mathfrak{c}$ is the quotient map, the kernel of $d \pi_{M}$ is just $\operatorname{Lie}(M) \cap$ $\operatorname{Lie}\left(C_{G}\left(K^{\circ}\right)\right) \subseteq \operatorname{Lie}(M) \cap \operatorname{Lie}\left(C_{G}\left(M^{\circ}\right)\right)$, and the latter is central in $\operatorname{Lie}(M)$. Then $\pi_{M}$ is central; cf. [9, Section 22.3]. Thus, by [9, 22.5 Corollary], the preimage of a $k$-defined torus is $k$-defined. Let $T=\lambda\left(\bar{k}^{*}\right)$ and let $S=\pi_{M}^{-1}(T)^{\circ}$. Thus $S$ and $T$ are 1-dimensional $k$-defined tori. Since $\pi$ is a $k$-morphism, it induces a map $Y_{k}(S) \rightarrow Y_{k}(T)$ whose image has finite index, say $n$. Then $\mu=n \lambda$ satisfies the required conditions.

The following is the rational version of Theorem 1 in the special case that $G=N$ and $C^{\circ}$ is a torus. The proof of Theorem 7.4 proceeds by reducing to this special case.

LEMMA 7.3. Let $G$ be a $k$-defined reductive group. Let $C$ be a $k$-defined normal subgroup of $G$ such that $C^{\circ}$ is a torus, and let $\pi$ be the quotient map $G \rightarrow G / C$. Let $M$ be a subgroup guaranteed by Lemma 7.1 so that $M \cap C$ is finite and $\pi$ induces an isogeny $\pi_{M}: M \rightarrow G / C$.

Then a subgroup $H$ of $G$ is $G$-completely reducible over $k$ if and only if $\pi(H)$ is $(G / C)$-completely reducible over $k$.

Proof. As in the proof of Theorem 1, it suffices to assume that $H \leqslant M$, since $H$ is contained in a parabolic subgroup $P_{\lambda}\left(\lambda \in Y_{k}(G)\right)$ if and only if $H C \leqslant P_{\lambda}$ if and only if $H C \cap M \leqslant P_{\lambda}$, and similarly for $L_{\lambda}$.

So suppose $H$ is $G$-cr over $k$, and suppose that $\pi(H) \leqslant P_{\mu}(G / C)$, where $\mu \in Y_{k}(G / C)$. By Lemma 7.2, we can assume that $\mu=\pi \circ \lambda$ for some $\lambda \in Y_{k}(M)$. Then $H \leqslant \pi^{-1}(\pi(H)) \leqslant \pi^{-1}\left(P_{\mu}\right)=P_{\lambda}(G)$, by Proposition 3.8. Since $H$ is $G$-cr over $k$, by [8, Lemma 2.5(iii)], there exists $u \in R_{u}\left(P_{\lambda}\right)(k)$ such that $H \leqslant L_{u \cdot \lambda}$. Now by [4, Lemma 6.15(iii)], we have $u \in R_{u}\left(P_{\lambda} \cap M\right)(k)$, so $u \cdot \lambda \in Y_{k}(M)$. Thus using Proposition 3.8 again, we have $\pi(H) \leqslant \pi\left(L_{u \cdot \lambda} \cap M\right)=L_{\pi(u) \cdot \mu}$. 
Moreover, $P_{\pi(u) \cdot \mu}=\pi\left(P_{u \cdot \lambda}\right)=\pi\left(P_{\lambda}\right)=P_{\mu}$, also by Proposition 3.8; so $L_{\pi(u) \cdot \mu}$ is an R-Levi subgroup of $P_{\mu}$. It follows that $\pi(H)$ is $(G / C)$-cr over $k$.

Conversely, suppose that $\pi(H)$ is $(G / C)$-cr over $k$, and suppose that $H \leqslant P_{\lambda}$ for some $\lambda \in Y_{k}(G)$. Then $\pi(H) \leqslant P_{\mu}$, where $\mu=\pi \circ \lambda \in Y_{k}(G / C)$. As $\pi(H)$ is $(G / C)$-cr over $k$, there is an R-Levi subgroup $L$ of $P_{\mu}$ such that $\pi(H) \leqslant L$. By the proof of Lemma 7.2, we can replace $\mu$ and $\lambda$ by some positive integer multiples (without changing the corresponding R-parabolic or R-Levi subgroups) so that there exists $\sigma \in Y_{k}(M)$ with $\pi \circ \sigma=\mu$. We have $\lambda\left(\bar{k}^{*}\right) \leqslant \sigma\left(\bar{k}^{*}\right) C^{\circ}$, and it follows that there exists a $k$-cocharacter $\tau \in Y_{k}(C)$ such that $\lambda=\sigma+\tau$.

Since $\pi(H)$ is $(G / C)$-cr over $k$, there exists $v \in Y_{k}(G / C)$ so that $P_{v}=P_{\mu}$ and $\pi(H) \leqslant L_{v}$. By Lemma 7.2, we can choose $v$ so that $v=\pi \circ \sigma^{\prime}$ for some $\sigma^{\prime} \in Y_{k}(M)$. Using Proposition 3.8 we have $P_{\sigma^{\prime}}=\pi^{-1}\left(P_{\nu}\right)=\pi^{-1}\left(P_{\mu}\right)=P_{\sigma}$. By [8, Corollary 2.6], we can adjust $v$ and $\sigma^{\prime}$ (without affecting the corresponding R-parabolic and R-Levi subgroups) so that $\sigma^{\prime}=u \cdot \sigma$ for some $u \in R_{u}\left(P_{\sigma} \cap\right.$ $M)(k)=R_{u}\left(P_{\sigma}\right)(k)=R_{u}\left(P_{\lambda}\right)(k)$. Thus, replacing $\lambda$ by $u \cdot \lambda$ if necessary, we can assume that $\pi(H) \leqslant L_{\mu}$. We have $H \leqslant \pi_{M}^{-1}\left(L_{\mu}\right)=L_{\sigma} \cap M$ by Proposition 3.8. We have $\lambda \in Y_{k}\left(L_{\sigma}\right)$, and

$$
H \leqslant P_{\lambda} \cap\left(L_{\sigma} \cap M\right) \leqslant P_{\lambda} \cap L_{\sigma}=P_{\tau} \cap L_{\sigma} .
$$

Now $L_{\sigma}^{\circ} \leqslant\left(L_{\sigma} \cap M^{\circ}\right) C^{\circ} \leqslant P_{\tau} \cap L_{\sigma}$; hence $R_{u}\left(P_{\tau} \cap L_{\sigma}\right)=\{1\}$, and so $P_{\tau} \cap L_{\sigma}=$ $L_{\tau} \cap L_{\sigma}$. Thus $H \leqslant L_{\tau} \cap L_{\sigma} \leqslant L_{\lambda}$, and so $H$ is $G$-cr over $k$.

The following is now the rational version of Theorem 1.

THEOREM 7.4. Let $K \leqslant G$ be reductive algebraic $k$-groups, write $N=N_{G}\left(K^{\circ}\right)$, $C=C_{G}\left(K^{\circ}\right)$, and let $\pi: N \rightarrow N / C$ be the quotient map. Suppose that $N$ and $C$ are $k$-defined, and let $H \leqslant N$.

Then $H$ is relatively $G$-completely reducible over $k$ with respect to $K$ if and only if $\pi(H)$ is $\pi(N)$-completely reducible over $k$.

Proof. The proof of Theorem 1 begins by reducing to the case that $G=N=$ $M Z\left(K^{\circ}\right)^{\circ}$, where $M$ is a reductive subgroup guaranteed by Lemma 3.1. Using Lemma 7.1, the same reduction holds in this rational setting, since none of the arguments require special properties of the field $k$, only that the groups and quotient maps involved are defined over $k$. So under this reduction, $Y_{k}(G)=$ $Y_{k}\left(G^{\circ}\right)=Y_{k}\left(K^{\circ}\right)=Y_{k}(K)$ so that $H$ is relatively $G$-cr over $k$ with respect to $K$ if and only if $H$ is $G$-cr over $k$. Moreover, after reduction to this case, the group $C^{\circ}=Z\left(K^{\circ}\right)^{\circ}$ is a torus, and so the required conclusion follows from Lemma 7.3. 
Remark 7.5. Note that while $G, K, N$ and $C$ all need to be $k$-defined for the above proofs to work, we do not make any such assumption on $H$.

7.2. Rational analogues of Theorem 3 and corollaries. In order to generalize Theorem 3 to arbitrary fields, we need a notion of a 'closed orbit' for a group $M(k)$ of $k$-points of a reductive $k$-group $M$ acting on a $k$-variety. The correct notion for us is as follows [8, Definition 3.8].

DEFINITION 5. Let $M$ be a reductive $k$-group and let $V$ be an $M$-variety defined over $k$. Let $v \in V$. We say that the $M(k)$-orbit $M(k) \cdot v$ is cocharacter-closed over $k$ if for any $\lambda \in Y_{k}(M)$ such that $v^{\prime}=\lim _{a \rightarrow 0} \lambda(a) \cdot v$ exists, $v^{\prime}$ is $M(k)$-conjugate to $v$.

The usefulness of this notion is shown by the following characterization of relative $G$-complete reducibility over $k$ in terms of cocharacter closure of a rational orbit of a generic tuple. Combining [3, Corollary 5.3], [7, Theorem 4.12 (iii)] and [3, Theorem 9.3], we obtain the following rational version of Theorem 2.1. For $K=G$, this is just [3, Theorem 9.3].

THEOREM 7.6. Let $K \leqslant G$ be reductive algebraic $k$-groups. Let $H$ be a subgroup of $G$ and let $\mathbf{h} \in H^{n}$ be a generic tuple of $H$. Then $H$ is relatively $G$-completely reducible over $k$ with respect to $K$ if and only if $K(k) \cdot \mathbf{h}$ is cocharacter-closed over $k$.

Owing to Theorem 7.6, Theorem 7.4 is equivalent to the following rational version of Theorem 3 .

THEOREM 7.7. Let $K \leqslant G$ be reductive algebraic $k$-groups, write $N=N_{G}\left(K^{\circ}\right)$, $C=C_{G}\left(K^{\circ}\right)$, and let $\pi: N \rightarrow N / C$ be the quotient map. Suppose that $N$ and $C$ are $k$-defined, let $\mathbf{h} \in N^{n}(n \geqslant 1)$ and write $\pi$ also for the map $N^{n} \rightarrow(N / C)^{n}$.

Then $K(k) \cdot \mathbf{h}$ is cocharacter-closed over $k$ if and only if $\pi(N)(k) \cdot \pi(\mathbf{h})$ is cocharacter-closed over $k$.

Next, Theorem 7.4 allows us to immediately deduce the relative version of [3, Corollary 9.7]. However, the result holds without the restriction $H \leqslant N$. One implication of the first part holds without the condition on $C_{K}(H)$, by [7, Theorem 4.13]. If $k^{\prime} / k$ is an algebraic extension of perfect fields, then both implications of the first part hold without the condition on $C_{K}(H)$, by [3, Theorem 4.14]. The next corollary therefore follows at once from [3, Theorem 5.7] and Theorem 7.6. 
COROLLARY 7.8. Let $K \leqslant G$ be reductive algebraic $k$-groups and let $H$ be a $k$-defined subgroup of $G$ such that $C_{K}(H)$ is $k$-defined. Then the following hold.

(1) For any separable algebraic extension $k^{\prime} / k, H$ is relatively $G$-completely reducible over $k^{\prime}$ with respect to $K$ if and only if $H$ is relatively $G$-completely reducible over $k$ with respect to $K$.

(2) For a $k$-defined torus $S$ of $C_{K}(H)$, let $L=C_{K}(S)$. Then $H$ is relatively $G$ completely reducible over $k$ with respect to $K$ if and only if $H$ is relatively $G$-completely reducible over $k$ with respect to $L$.

We end this section with a geometric consequence of Theorem 7.7, which provides a rational version of Theorem 4.12. For that, we require the notion of a cocharacter-closed subset of a $G$-variety over $k$ [3, Definition 1.2(a)].

DEFINITION 6. Let $V$ be an affine variety over $k$ on which $G$ acts. Given a subset $X$ of $V$, we say that $X$ is cocharacter-closed (over $k$ ) if for every $v \in X$ and every $\lambda \in Y_{k}(G)$ such that $v^{\prime}=\lim _{a \rightarrow 0} \lambda(a) \cdot v$ exists, $v^{\prime} \in X$.

Note that this definition coincides with Definition 5 if $X=G(k) \cdot v$ for some $v \in V$.

The cocharacter-closed subsets of $V$ form the closed sets of a topology on $V$ (it is clear that arbitrary intersections and unions of cocharacter-closed sets are cocharacter-closed, and that the empty set and the whole space $V$ are cocharacterclosed); cf. [3, Remark 3.1(iii)]. It is this topology that is used in our rational version of Theorem 4.12 .

THEOREM 7.9. Let $K \leqslant G$ be reductive algebraic $k$-groups, write $N=N_{G}\left(K^{\circ}\right)$, $C=C_{G}\left(K^{\circ}\right)$, and let $\pi: N \rightarrow N / C$ be the quotient map. Let $\mathbf{h} \in N^{n}(n \geqslant 1)$ and write $\pi$ also for the map $N^{n} \rightarrow(N / C)^{n}$. Suppose that $N$ and $C$ are $k$-defined. Then the following are equivalent.

(1) $K(k) \cdot \mathbf{h}$ is cocharacter-closed over $k$ in $G^{n}$.

(2) $\pi(K(k)) \cdot \pi(\mathbf{h})$ is cocharacter-closed over $k$ in $(N / C)^{n}$.

(3) $\pi^{-1}(\pi(K(k) \cdot \mathbf{h}))$ is cocharacter-closed over $k$ in $G^{n}$.

(4) Every $K(k)$-orbit on $\pi^{-1}(\pi(K(k) \cdot \mathbf{h}))$ is cocharacter-closed over $k$ in $G^{n}$.

Proof. Thanks to Theorem 7.7, (1) and (2) are equivalent. Since $N^{n}$ is closed in $G^{n}$ and stabilized by $K$, it is cocharacter-closed for the action of $K$ by [3, Remark 3.1(ii)]. Further, since $\pi(K(k)) \cdot \pi(\mathbf{h})=\pi(K(k) \cdot \mathbf{h})$ and the topology 
on $(N / C)^{n}$ is the quotient topology afforded by the cocharacter-closed subsets of $N$, it follows that (2) and (3) are equivalent.

Clearly, (4) implies (1). Now suppose (1). Thanks to [3, Corollary 3.5], $K(k) \cdot \mathbf{h}$ is cocharacter-closed over $k$ if and only if $K^{\circ}(k) \cdot \mathbf{h}$ is cocharacter-closed over $k$. Thus we may assume that $K$ is connected. Writing $\mathbf{h}=\left(h_{1}, \ldots, h_{n}\right)$, we have

$$
\begin{aligned}
\pi^{-1}(\pi(K(k) \cdot \mathbf{h})) & =\left\{\left(\left(x \cdot h_{1}\right) c_{1}, \ldots,\left(x \cdot h_{n}\right) c_{n}\right): x \in K(k), c_{i} \in C\right\}, \\
& =\left\{\left(x \cdot\left(h_{1} c_{1}\right), \ldots, x \cdot\left(h_{n} c_{n}\right)\right): x \in K(k), c_{i} \in C\right\},
\end{aligned}
$$

where the last equality follows again since $K=K^{\circ}$ centralizes $C$. Thus $\pi^{-1}(\pi(K(k) \cdot \mathbf{h}))$ is a union of $K(k)$-orbits, each of which is a translate of the cocharacter-closed $K(k)$-orbit $K(k) \cdot \mathbf{h}$ by an element of $C^{n}$. As translation is a $k$-variety automorphism $G^{n} \rightarrow G^{n}$, it follows that every $K(k)$-orbit on $\pi^{-1}(\pi(K(k) \cdot \mathbf{h}))$ is cocharacter-closed in $G^{n}$, as desired.

\section{Acknowledgements}

The second author acknowledges support from the Alexander von Humboldt Foundation. The authors wish to thank Ben Martin for helpful comments on early versions of the paper.

Conflict of Interest: The authors declare there are no conflicts of interest.

\section{References}

[1] C. Attenborough, M. Bate, M. Gruchot, A. Litterick and G. Röhrle, 'On relative complete reducibility', Q. J. Math. 71 (2020), 321-334.

[2] M. Bate, S. Herpel, B. Martin and G. Röhrle, ' $G$-complete reducibility and semisimple modules', Bull. Lond. Math. Soc. 43(6) (2011), 1069-1078.

[3] M. Bate, S. Herpel, B. Martin and G. Röhrle, 'Cocharacter-closure and the rational HilbertMumford theorem', Math. Z. 287(1-2) (2017), 39-72.

[4] M. Bate, B. Martin and G. Röhrle, 'A geometric approach to complete reducibility', Invent. Math. 161(1) (2005), 177-218.

[5] M. Bate, B. Martin and G. Röhrle, 'Complete reducibility and commuting subgroups', J Teine Angew. Math. (Crelles J.) 2008(621) (2008), 213-235.

[6] M. Bate, B. Martin, G. Röhrle and R. Tange, 'Complete reducibility and separability', Trans. Amer. Math. Soc. 362(08) (2010), 4283-4311.

[7] M. Bate, B. Martin, G. Röhrle and R. Tange, 'Complete reducibility and conjugacy classes of tuples in algebraic groups and Lie algebras', Math. Z. 269(3-4) (2011), 809-832.

[8] M. Bate, B. Martin, G. Röhrle and R. Tange, 'Closed orbits and uniform $S$-instability in geometric invariant theory', Trans. Amer. Math. Soc. 365(7) (2013), 3643-3673.

[9] A. Borel, Linear Algebraic Groups, 2nd edn, Graduate Texts in Mathematics, 126 (Springer, New York, 1991). 
[10] M. Brion, 'On extensions of algebraic groups with finite quotient', Pacific J. Math. 279(1-2) (2015), 135-153.

[11] J. C. Jantzen, Low Dimensional Representations of Reductive Groups are Semisimple, Algebraic Groups and Lie Groups (Cambridge University Press, Cambridge, England, 1997).

[12] M. W. Liebeck and G. M. Seitz, 'Reductive subgroups of exceptional algebraic groups', Mem. Amer. Math. Soc. 121(580) (1996), pp. vi+111.

[13] A. J. Litterick and A. R. Thomas, 'Complete reducibility in good characteristic', Trans. Amer. Math. Soc. 370(8) (2018), 5279-5340.

[14] G. McNinch, 'Dimensional criteria for semisimplicity of representations', Proc. Lond. Math. Soc. 76 (1998), 95-149.

[15] G. McNinch, 'Completely reducible Lie subalgebras', Transform. Groups 12(1) (2007), 127-135.

[16] R. W. Richardson, 'On orbits of algebraic groups and Lie groups', Bull. Austr. Math. Soc. 25 (1982), 1-28.

[17] J.-P. Serre, Moursund lectures, University of Oregon, arXiv:math/0305257, 1998.

[18] J.-P. Serre, 'Complète réductibilité', Séminaire Bourbaki 46(2003-2004) 195-218; (in French).

[19] T. A. Springer, Linear Algebraic Groups, 2nd edn, Modern Birkhäuser Classics (Birkhäuser Boston, Inc., Boston, MA, 2009).

[20] D. I. Stewart, 'Non- $G$-completely reducible subgroups of the exceptional algebraic groups', Int. Math. Res. Not. IMRN 2014(22) (2013), 6053-6078.

[21] T. Uchiyama, 'Non-separability and complete reducibility: $E_{n}$ examples with an application to a question of Külshammer', J. Group Theory 20(5) (2017). 